Check for updates

Cite this: RSC Adv., 2019, 9, 9809

\title{
Diastereoselective approach to rationally design tetrahydro- $\beta$-carboline-isatin conjugates as potential SERMs against breast cancer $\dagger$
}

\author{
Bharvi Sharma, ${ }^{a}$ Amandeep Singh, ${ }^{a}$ Liang Gu, ${ }^{b}$ Sourav Taru Saha, ${ }^{\text {b }}$ Ashona Singh- \\ Pillay, ${ }^{c}$ Nosipho Cele, ${ }^{c}$ Parvesh Singh, ${ }^{c}$ Mandeep Kaur ${ }^{\star b}$ and Vipan Kumar (D) *a
}

\begin{abstract}
A series of tetrahydro- $\beta$-carboline-isatin conjugates, with varying substituents as well as stereochemistry at C-1 and $\mathrm{C}-5$ position of tetrahydro- $\beta$-carboline $(\mathrm{TH} \beta \mathrm{C})$ and isatin ring, were prepared and assayed for antiproliferative efficacy on Estrogen Responsive ER(+) (MCF-7) and ER(-ve) MDA-MB-231 cell-lines. The synthesized scaffolds displayed selective anti-proliferative efficacy against MCF-7 cell-line with the most active conjugate $8 \mathrm{~b}$ exhibiting an $\mathrm{IC}_{50}$ value of $37.42 \mu \mathrm{M}$, comparable to that of peganumine $A$, a tetrahydro- $\beta$-carboline analogue, isolated from Peganum harmala. The synthesized compound $8 \mathrm{~b}$ was also more potent than the standard drug tamoxifen $\left(\mathrm{IC}_{50}=50 \mu \mathrm{M}\right.$ against MCF-7). The observed activities were further corroborated via docking studies in ER- $\alpha$ (PDB ID: 3ERT).
\end{abstract}

Received 28th January 2019 Accepted 11th March 2019

DOI: $10.1039 / c 9 r a 00744 j$

rsc.li/rsc-advances

anti-proliferative activity against MDA-MB-231 cell-lines with $\mathrm{IC}_{50}$ values in the $\mu \mathrm{M}$ range. ${ }^{9}$ The anti-proliferative potential of TH $\beta$ Cs along with their low cytotoxicity, attributed to the lack of DNA intercalating properties owing to its non-planar structure, has recently attracted the attention of the scientific fraternity for the development of selective anti-proliferatives. ${ }^{8}$

Isatin is another indole-based moiety with proven antiproliferative potential via inhibition of tyrosine kinases like VEGFR-1, VEGFR-2 and cyclic dependent kinases (CDKs). ${ }^{\mathbf{1 0}}$ Sutent ${ }^{\circledR}$, an isatin analogue (5-fluoro-3-alkenyl-2 oxindole) has been approved by the FDA for the treatment of gastrointestinal stromal tumors and advanced renal cell carcinoma. ${ }^{11}$ Although market is saturated with first line drugs and potential SERMS sanctioned by US Food and Drug Administration (FDA) viz. tamoxifen (TAM), raloxifene having significant anti-BC activities, yet they are also associated with adverse side effects such as endometrial cancer, insomnia, dizziness. ${ }^{\mathbf{1 2}}$ Recent reports from our lab have shown in vitro anti-proliferative activities of ospemifene-isatins against breast cancer cell lines. The inclusion of isatin ring among these conjugates enhanced the antiproliferative activities in general with the most potent conjugate exhibiting an $\mathrm{IC}_{50}$ of $1.56 \mu \mathrm{M}$ against MCF-7, being $\sim 30$ folds potent than tamoxifen. ${ }^{13}$ The methodology was further extended towards evaluating the anti-proliferative efficacy of $1 H$-1,2,3-triazole linked nitro-imidazole-isatin and isatin-ferrocenyl chalcone conjugates. ${ }^{\mathbf{1 4 , 1 5}}$

In continuation to our recent reports on the synthesis of molecular conjugates with biological relevance and their evaluation, ${ }^{\mathbf{1 6 - 1 8}}$ the present manuscript encompasses the design, synthesis and anti-proliferative evaluation of $1 H$-1,2,3-triazole linked $\mathrm{TH} \beta \mathrm{C}$-isatin conjugates, as depicted in Fig. 2, against MCF-7 and MDA-MB-231 cell-lines using MTT assay. The use of 

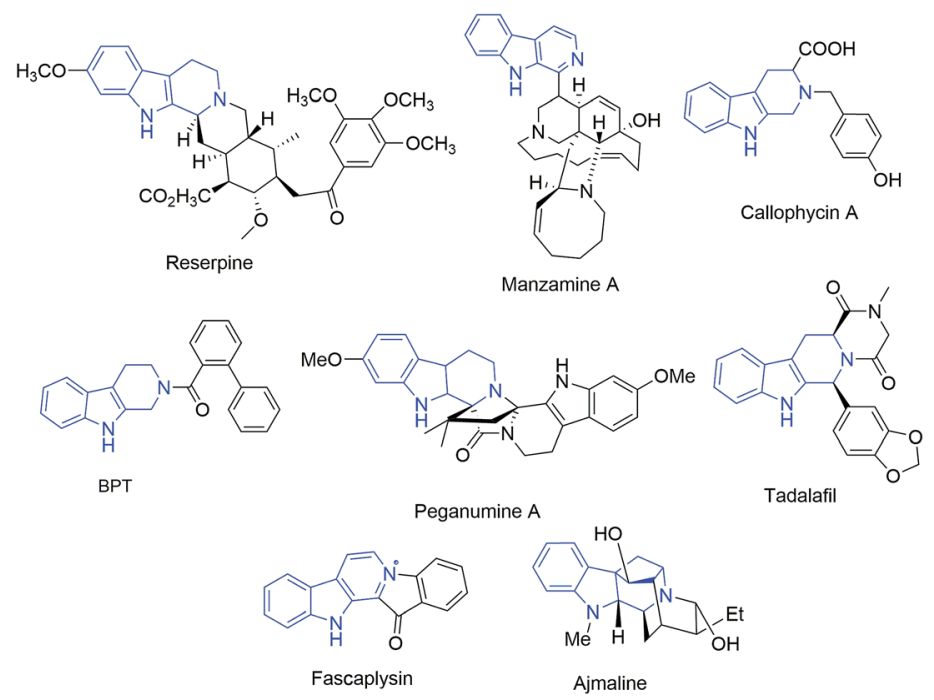

Fig. 1 Natural and synthetic structural motifs with a tetrahydro- $\beta$-carboline core.
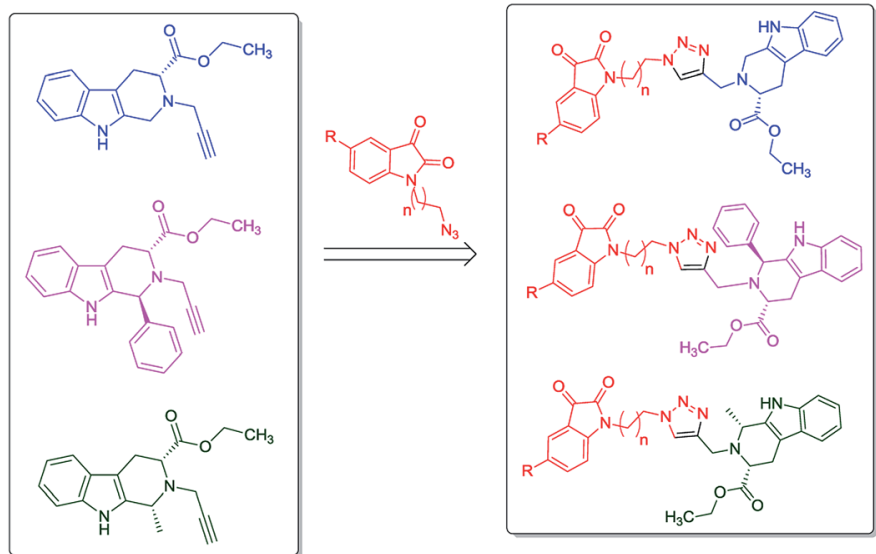

Fig. 2 Designed tetrahydro- $\beta$-carboline-isatin conjugates.

L-tryptophan in the present series of compounds is not only owed to its natural availability but also because of the prom-

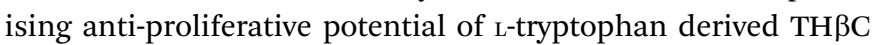
against MDA-MB-231 cell-line. The nature of substituents as well as the stereochemistry at C- 1 of $\mathrm{TH} \beta \mathrm{C}$ and C- 5 of isatin ring was varied so as to study their effect on the anti-proliferative activities.

\section{Results and discussion}

\section{Chemistry}

The synthetic methodology involved the enantioselective Pictet-

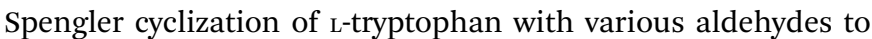
afford the corresponding tetrahydro- $\beta$-carbolines with varying stereochemistry at C-1 position. ${ }^{19,20}$ Depending upon the reactivity of different aldehydes, different reaction conditions were employed for the synthesis of corresponding TH $\beta \mathrm{C}$ derived carboxylic acids. The preparation of 2,3,4,9-tetrahydro- $1 H$ - $\beta$ carboline-3-carboxylic acid $\mathbf{2 a}$ involved the base-promoted
Pictet-Spengler reaction of L-tryptophan with 30\% aqueous solution of formalin at room temperature. In the case of aliphatic aldehydes viz. acetaldehyde, a kinetically controlled Pictet-Spengler reaction of $\mathrm{L}_{\mathrm{t}}$-tryptophan with $40 \%$ solution of acetaldehyde yielded cis-isomer( $1 S, 3 S)$-1-methyl-2,3,4,9-tetrahydro- $1 H$-pyrido-[3,4- $\beta]$ indole-3-carboxylic acid $\mathbf{2 b}$ as the major product.

With a less reactive aromatic aldehyde viz. benzaldehyde, the reaction upon heating in acetic acid followed a thermodynamically controlled pathway to afford a diastereomeric mixture which was used in subsequent steps without purification. The treatment of TH $\beta \mathrm{C}$ derived acids $\mathbf{2 a - c}$ with thionyl chloride in dry ethanol afforded the corresponding esters 3a-c. Compound $3 \mathbf{c}$ having the phenyl substituent at C-1 was successfully purified using column chromatography and only the trans isomer was used in subsequent steps because of its prevalence in naturally occurring alkaloids viz. ajmaline, ${ }^{21}$ sarpagine ${ }^{22}$ as well as the non-natural analogue tadalafil. Base-promoted $N$-propargylation of 3a-c in dry acetonitrile resulted in the formation of the corresponding 'click' precursors 4a-c (Scheme 1). The second precursor viz. $N$-alkylazidoisatins 7 was obtained via base-promoted alkylation of C-5 substituted isatin 5 with dibromoalkane to afford $\mathbf{6}$ with successive treatment with sodium azide (Scheme 2).

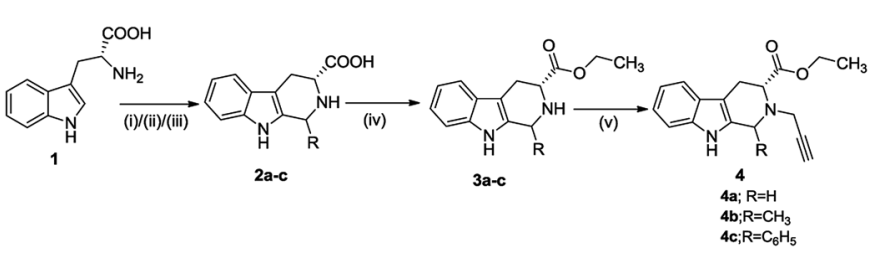

Scheme 1 Synthesis of $N$-propargylated tetrahydro- $\beta$-carboline ethyl ester. (i) $30 \%$ formaldehyde, $\mathrm{NaOH}, \mathrm{H}_{2} \mathrm{O}, 8 \mathrm{~h}$, reflux, or (ii) $40 \%$ acetaldehyde, $\mathrm{H}_{2} \mathrm{SO}_{4}, 6-8 \mathrm{~h}$, rt, or (iii) benzaldehyde, acetic acid, $2 \mathrm{~h}$, reflux (iv) $\mathrm{SOCl}_{2}, \mathrm{EtOH}, 6 \mathrm{~h}$, reflux (v) propargyl bromide, acetonitrile, rt, 6$8 \mathrm{~h}$. (vi) $\mathrm{CuSO}_{4} \cdot 5 \mathrm{H}_{2} \mathrm{O}$, sodium ascorbate, $\mathrm{EtOH}$, rt, $8 \mathrm{~h}$. 
<smiles>[R]c1ccc2c(c1)C(=O)C(=O)N2</smiles><smiles>[R]c1ccc2c(c1)C(=O)C(=O)N2C(C)C</smiles><smiles>[R]c1ccc2c(c1)C(=O)C(=O)N2CCN</smiles>

Scheme 2 Synthesis of substituted $\mathrm{N}$-alkyl-azido-isatin (i) $\mathrm{NaH}$, dibromoalkane, anhy. DMF, $60{ }^{\circ} \mathrm{C}, 6-8 \mathrm{~h}$ (ii) $\mathrm{NaN}_{3}, \mathrm{DMF}, 60^{\circ} \mathrm{C}, 2 \mathrm{~h}$.

$\mathrm{Cu}$-promoted azide-alkyne cycloaddition reactions of $\mathbf{4 a - c}$ with 7a-f gave the desired TH $\beta \mathrm{C}$-isatin conjugates $\mathbf{8 a - r}$ in good yields as shown in Scheme 3. The compounds thus synthesized were purified using ethylacetate : hexane $(80: 20)$ on alumina as a stationary phase.

The structure to the compounds was assigned on the basis of analytical evidences and spectral data. For example, the most active compound 2-\{1-[3-(2,3-dioxo-2,3-dihydro-indol-1-yl)propyl]-1 $H$-[1,2,3]triazol-4-ylmethyl $\}-2,3,4,9$-tetrahydro- $1 H$ - $\beta$ carboline-3-carboxylic acid ethyl ester (8b) displayed molecular ion peak $m / z$ at $[\mathbf{M}+1]^{+} 513.2206$ in its High Resolution Mass Spectrum (HRMS). The prominent features in its ${ }^{1} \mathrm{H}$ NMR include the appearance of triplet at $\delta 1.23$ corresponding to methyl protons, multiplet corresponding to methylene ( $\mathrm{H}-4)$ of TH $\beta$ C ring at $\delta 3.09-3.21$ and singlet because of triazole- $\mathrm{H}$ at $\delta$ 7.67. Further, ${ }^{13} \mathrm{C}$ NMR confirmed the assigned structure by the appearance of characteristic absorptions at $\delta 172.7$ representing the carbonyl carbon of TH $\beta \mathrm{C}$ along with $\delta 158.5$ and 183.0 corresponding to isatin ring carbonyls.

\section{Anti-proliferative activities of synthesized conjugates}

The newly synthesized conjugates were screened for their antiproliferative activities against human breast cancer cell-lines viz. MCF-7 and MDA-MB-231 and the results were compared with reference drugs, plumbagin ${ }^{23}$ and peganumine A. ${ }^{7}$ Detailed results of these studies, in terms of \% age growth inhibition of MCF-7 and MDA-MB-231 cells, have been depicted in Fig. 3. The

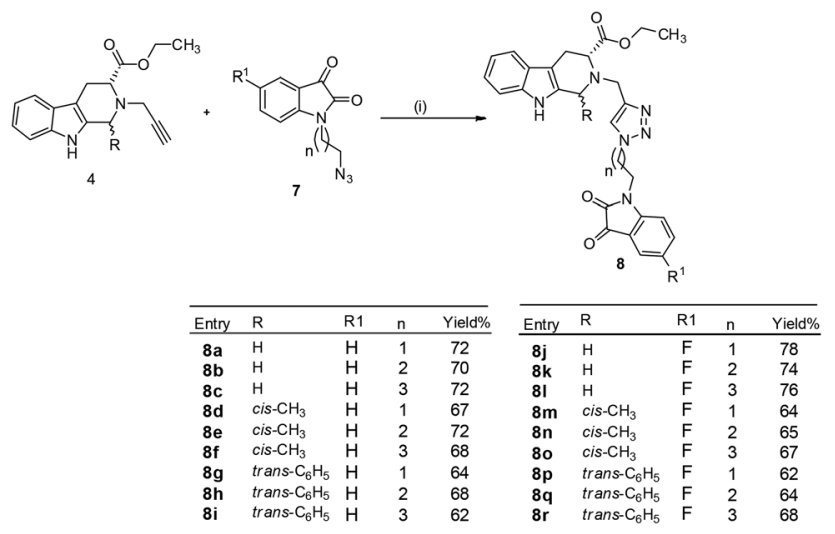

Scheme 3 Synthesis of $1 \mathrm{H}-1,2,3$-triazole-linked tetrahydro- $\beta$-carboline-isatin conjugates $8 \mathrm{a}-\mathrm{r}$ (i) $\mathrm{CuSO}_{4}$, sodium ascorbate, $\mathrm{EtOH}, 6-8 \mathrm{~h}$, rt.
$\mathrm{IC}_{50}$ value i.e. the necessary concentration for inhibiting $50 \%$ of cell viability by the synthesized compounds, has been enlisted in Table 1.

As evident from Table 1, all the synthesized conjugates proved to be inactive against MDA-MB-231 (ER-) cell-line and were selective towards estrogen responsive MCF-7 cell-line. Structure activity relationship analysis showed the dependence of anti-proliferative activity on the nature of substituent at $\mathrm{C}-1$ and $\mathrm{C}-5$ position of $\mathrm{TH} \beta \mathrm{C}$ and isatin ring respectively while the alkyl chain length did not seem to play any substantial role. Among un-substituted $(\mathrm{R}=\mathrm{H})$ isatin based conjugates $\mathbf{8 a}-$ $\mathbf{8 i}$, the presence of either hydrogen or methyl substituents at C-1 position of $\mathrm{TH} \beta \mathrm{C}$ resulted in the loss of activities as evident from $8 \mathbf{g}-\mathbf{8 i}$. The induction of fluoro-substituent at C-5 position of isatin ring proved to be detrimental for anti-proliferative activities as most of the compounds were inactive except 8r, having a phenyl substituent at $\mathrm{C}-1$, exhibiting an $\mathrm{IC}_{50}$ value of 42.11 $\mu \mathrm{M}$. Interestingly, several compounds (8k-n) showed a negative correlation, where an increase in drug concentration induced a decrease in growth inhibition, possibly suggesting that these compounds are supporting cancer cell proliferation. However, it could also suggest that at high concentrations, these compounds might have precipitated out of culture media and thus lost their effectiveness.

\section{Molecular docking studies}

In order to identify novel binding cores, molecular docking of the active ligands in the binding site of estrogen receptor- $\alpha$ (ER$\alpha$ ) was performed to determine the crucial binding interactions responsible for the inhibitory effect observed. ${ }^{24}$ The native ligand in the crystal structure of ER- $\alpha$ (PDB ID: 3ERT) is afimoxifene which inhibited the receptor by binding to the predominantly hydrophobic pocket. The binding interaction profile of the ligand-protein complex largely comprised of hydrophobic interactions with residues Leu41, Ala45, Trp78, Leu82, Leu86, Phe99, Leu220 and Leu231. These interactions were accompanied by two distinct hydrogen bonds with Glu48 and Arg89, two water bridges established between residues Thr42 and Leu86 as well as a single salt bridge with amino acid Asp46.

Compound $\mathbf{8 b}$ shared a similar hydrophobic interaction network to that of the native ligand. The binding landscape of compound $\mathbf{8 b}$ comprised a significant hydrophobic contribution between residues, Leu41, Leu44, Ala45, Leu49, Trp78, Leu82, Leu220 and Leu231 with the phenyl fused heterocyclic rings and the hydrophobic linker (bond distance $=3.93 / 3.77$, 3.99, 3.81, 3.78, 3.62/3.87, 3.95, 3.95 and $3.84 \AA$ A, respectively). A single salt bridge exists between the positively charged tertamine and Asp46 at a distance of 5.11 A. Despite the binding profile of compound $\mathbf{8 b}$ being very simple, it was identified as the most potent inhibitor (Fig. 4).

Compounds 8d, 8e and 8r demonstrated promising activity against the MCF-7 cell-line in comparison to peganumine A. Compounds $\mathbf{8 d}$ and $\mathbf{8 e}$ have a near identical binding interaction profile. The binding of these compounds to the active site of ER$\alpha$ comprised an extensive network of van der Waals interactions 

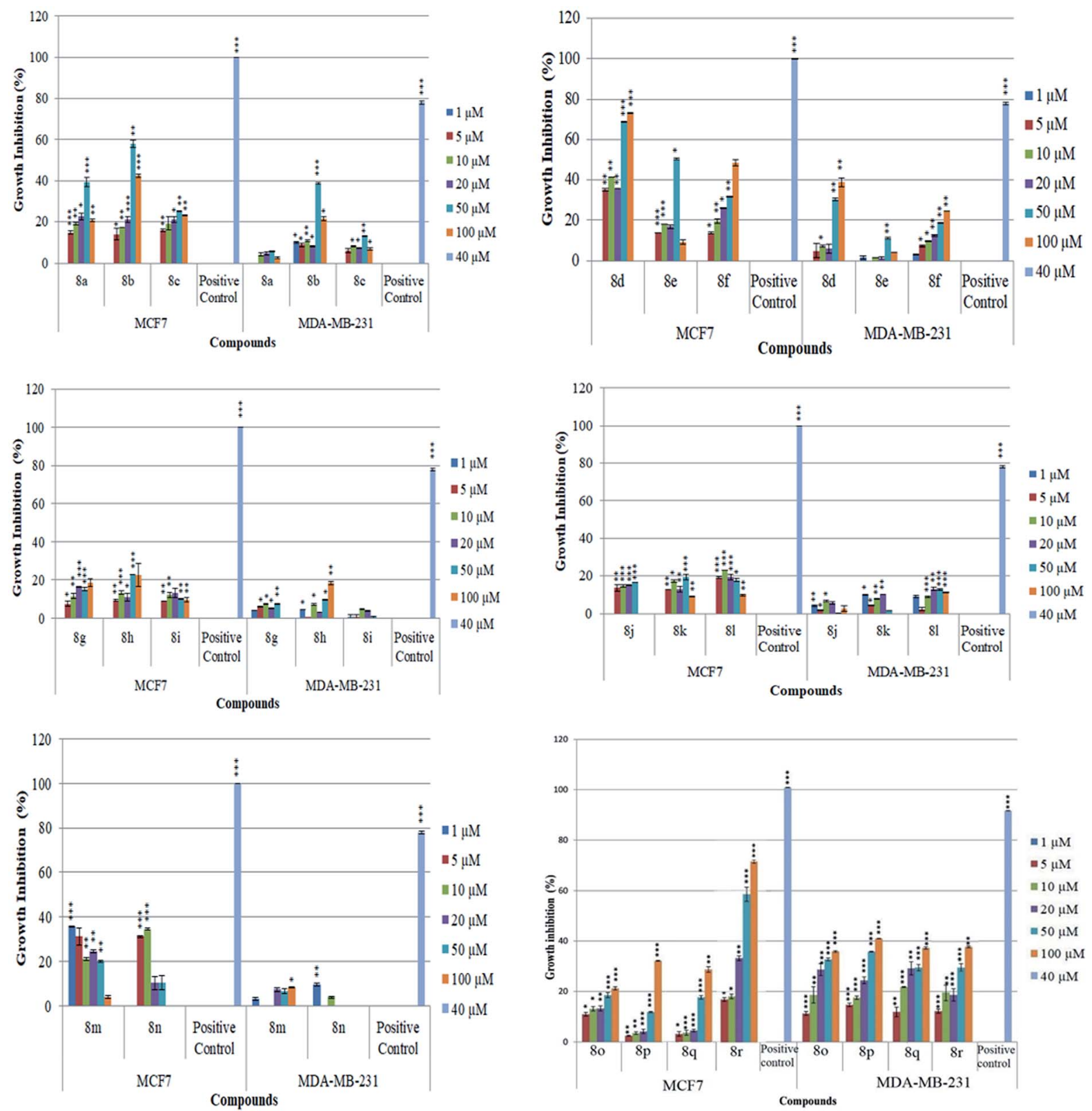

Fig. 3 Representative graph of percentage growth inhibition on MCF7 and MDA-MB-231 cells at selected concentrations of test compounds $8 a-r .40 \mu$ M plumbagin was used as a positive control. Data shows mean \pm standard deviation S.D. $(n=3)$, where $* p<0.05, * * p<0.01$ and $* * * p<$ 0.001 significance compared to untreated control.

between residues Leu41, Leu44, Ala45, Leu49, Trp78, Leu86, Phe99, Lys224 and Leu231 and the aromatic moieties of the ligands (bond distance $=4.00 / 3.90 / / 3.92 / 3.78,3.97,3.68 / / 3.76$, $3.83,3.83 / / 3.54,3.87,3.83,3.96 / 3.95 / / 3.39$ and 3.85//3.82 $\AA$; respectively). The protein-ligand interaction was further supported by a hydrogen bond between the oxygen of the hydrogen acceptor Leu220 and the triazole nitrogens (bond distance $=$ $3.00 / / 3.54 \AA$; bond angle $=136.41^{\circ} / / 105.19^{\circ}$ ) as well as a salt bridge between the anion Asp46 and the tertamine (bond distance $=4.27 / / 5.06 \AA$ ).

The inhibitory activity of compound $\mathbf{8 r}$ was largely driven by the existence of the hydrophobic network between residues
Leu41, Ala45, Trp78, Leu82, Leu86, Tyr221 and Leu231 at a bond distance of 3.70, 3.75, 3.64, 3.80/3.87, 3.52, 3.99 and 3.69 $\AA$, respectively. Also, the ligand interaction included two hydrogen bonds between the donor residue Asp46 and the carbonyl oxygen of the ligand ethoxy group as well as the acceptor residue Leu220 and the aromatic nitrogen (bond distance $=3.82$ and $2.93 \AA$; bond angle $=100.07^{\circ}$ and $166.19^{\circ}$; respectively). The fluorine moiety of compound $8 \mathbf{r}$ also formed a distinct electrostatic interaction with residue Arg89, (bond distance $=3.18 \AA$, donor angle $=141.31^{\circ}$, acceptor angle $=$ $\left.109.50^{\circ}\right)$. The thermodynamic and conformational stability of the complexes $\mathbf{8 b}, \mathbf{8 d}, \mathbf{8 e}$ and $\mathbf{8 r}$ in the binding site has been well 
View Article Online

Paper

RSC Advances

Table $1 \quad I C_{50}$ values of the test compounds in MCF-7 and MDA-MB-231 cells

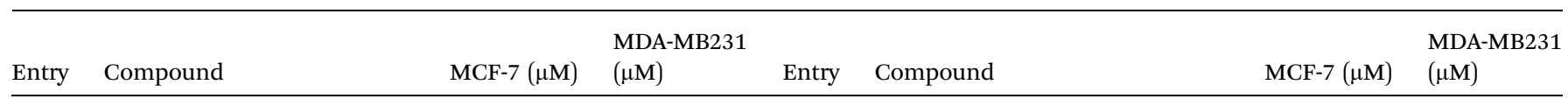

$8 a$

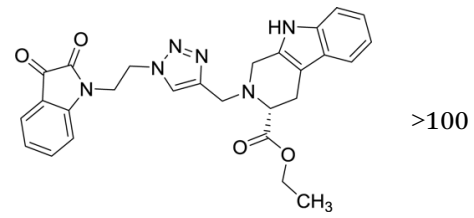

$8 b$

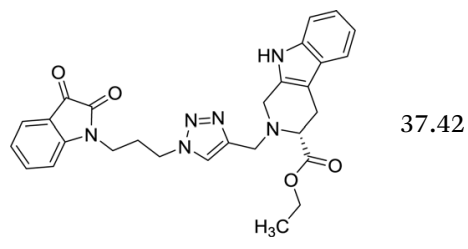

$8 c$

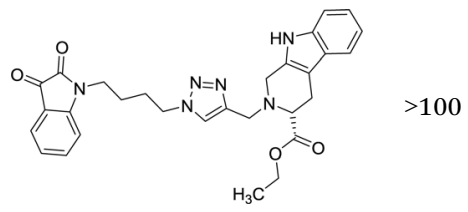

Bd

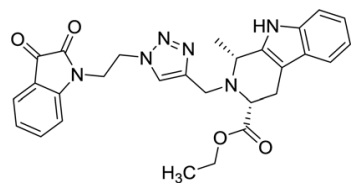

45.3

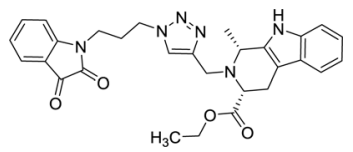

50

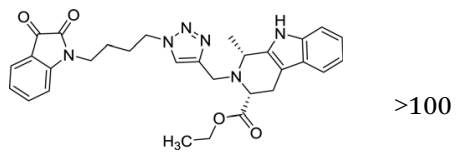

89

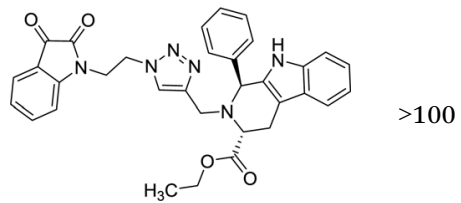

$8 h$

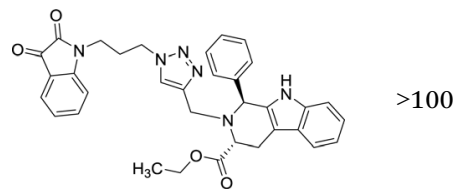

$8 i$

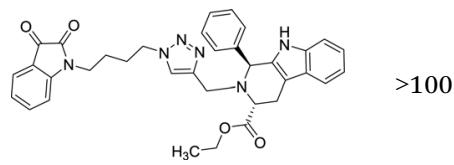

$8 \mathbf{j}$

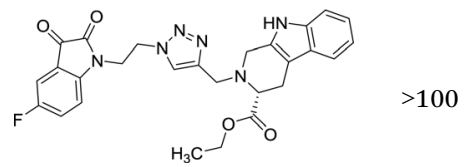

$>100$

sk

$>100$

81

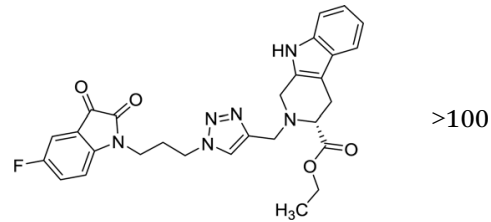

$8 m$

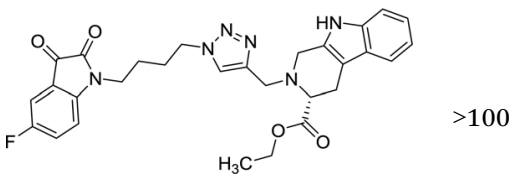

$>100$

$>100$

$8 n$
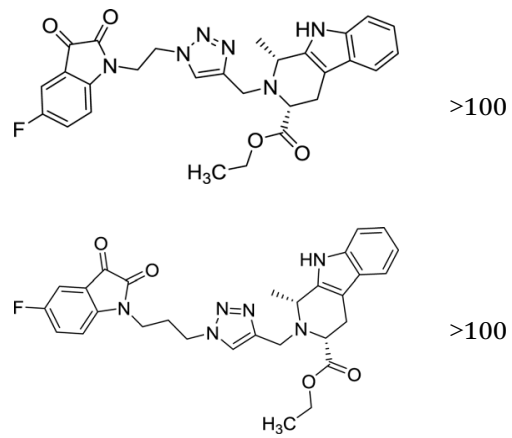

80

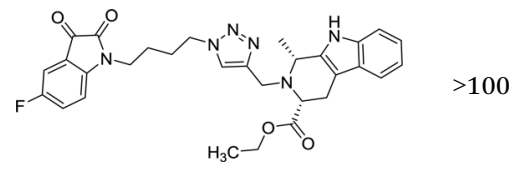

sp

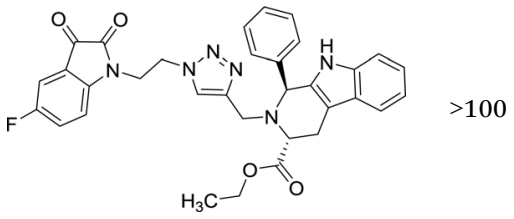

$8 q$

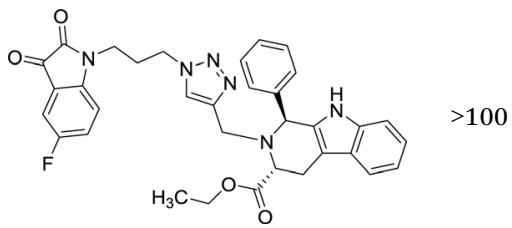

$8 r$

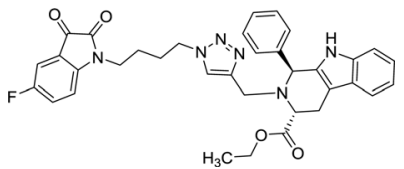

$>100$

Plumbagin

Peganumine $\mathrm{A}^{7}$ Tamoxifen
$>100$

42.11

$>100$

3.5

$>100$

$>100$

$>100$

$>100$

$>100$

$>100$

$>100$

4.4

38.5

50

75

This journal is (c) The Royal Society of Chemistry 2019

RSC Adv., 2019, 9, 9809-9819 | 9813 


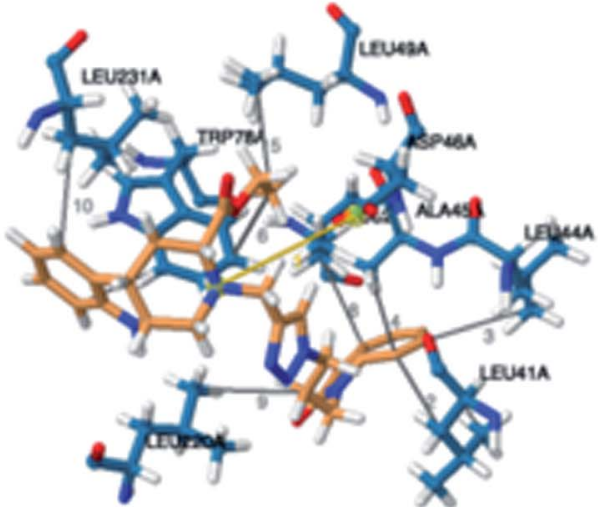

Compound 8b

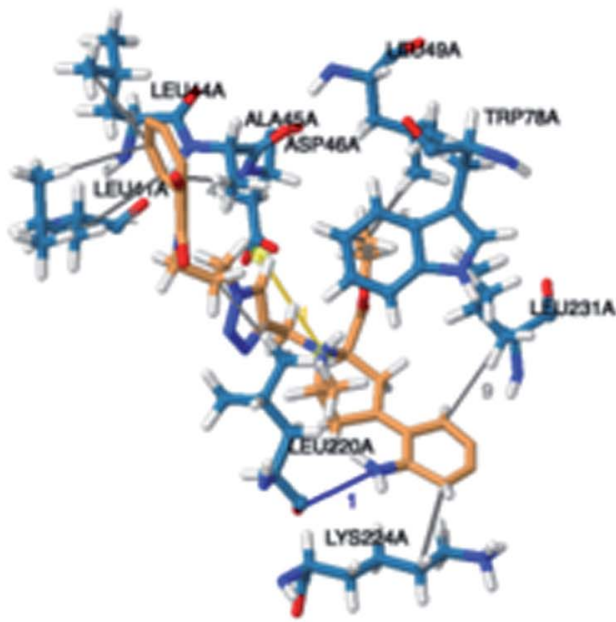

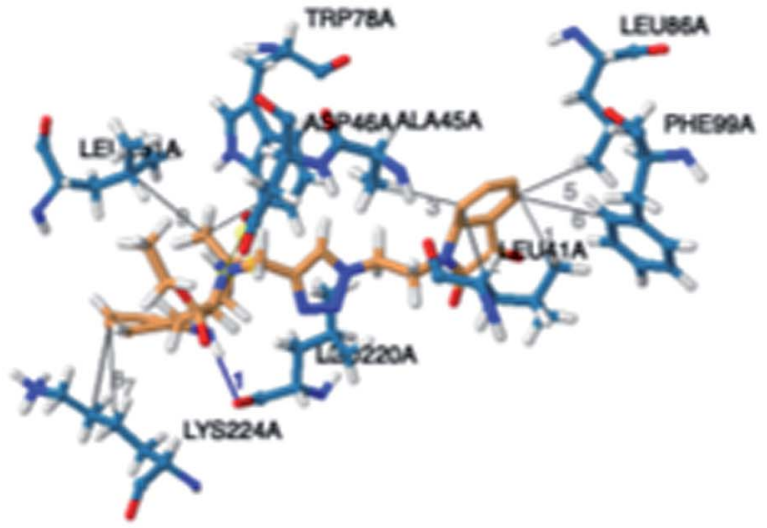

Compound $8 \mathrm{~d}$

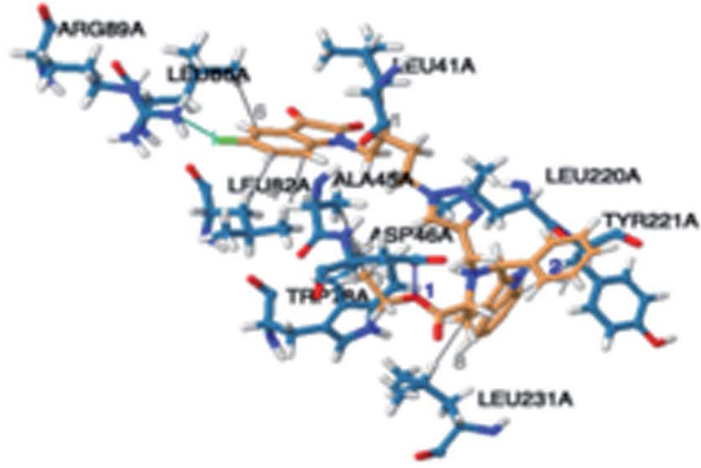

Compound $8 \mathrm{r}$

\section{Compound $8 \mathrm{e}$}

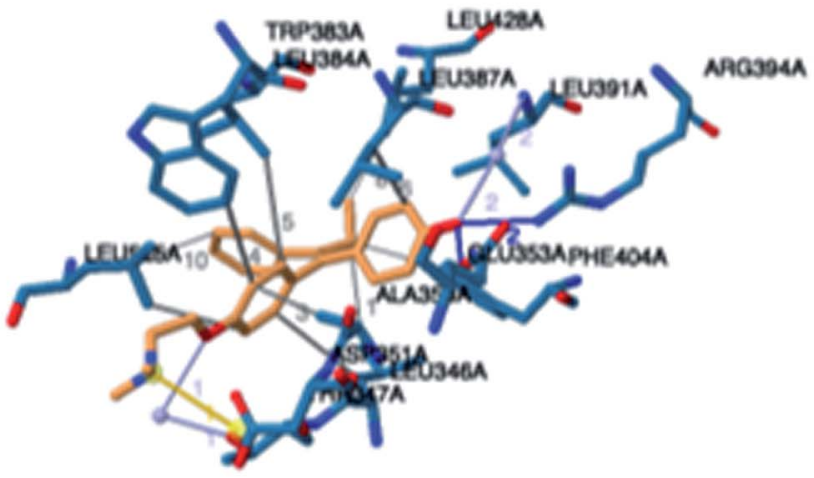

\section{Native ligand}

Fig. 4 Three-dimensional illustration of docked ligands interacting in the binding site of the estrogen receptor (PDB: 3ERT).

depicted by high binding affinities with energy value of -9.6 , $-8.8,-9.9$ and $-10.5 \mathrm{kcal} \mathrm{mol}^{-1}$ respectively (Table 2). The parameters describing the physicochemical properties viz. $\log \mathrm{P}$ and topological polar surface area (TPSA) of the target compounds have been well demonstrated in Table 3 (see ESI $\dagger$ ). The synthesized conjugates $\mathbf{8 b}, \mathbf{8 d}, \mathbf{8 e}$ and $\mathbf{8 f}$ (TPSA/ $\AA$ = 110.01, $110.01,110.01$ and 110.10 respectively) possess good potential to permeate cell membrane. Further, the moderate positive 
Table 2 Docking of the most active compounds in ER- $\alpha$

\begin{tabular}{llll}
\hline & $\begin{array}{l}\text { Docking score/(kcal } \\
\text { Ligand identifier }^{-1} \text { ) }\end{array}$ & RMSD i.b. & RMSD u.b. \\
\hline 8a & -9.7 & 0.000 & 0.000 \\
8b & -9.6 & 0.000 & 0.000 \\
$\mathbf{8 c}$ & -9.7 & 0.000 & 0.000 \\
$\mathbf{8 d}$ & -8.8 & 1.960 & 2.689 \\
$\mathbf{8 e}$ & -9.9 & 0.000 & 0.000 \\
$\mathbf{8 f}$ & -8.7 & 1.978 & 2.557 \\
$\mathbf{8 g}$ & -9.3 & 2.080 & 2.582 \\
$\mathbf{8 h}$ & -9.6 & 2.577 & 4.014 \\
$\mathbf{8 i}$ & -10.4 & 0.000 & 0.000 \\
$\mathbf{8 j}$ & -10.2 & 0.000 & 0.000 \\
$\mathbf{8 k}$ & -10.2 & 0.000 & 0.000 \\
$\mathbf{8 1}$ & -9.3 & 1.193 & 1.427 \\
$\mathbf{8 m}$ & -9.5 & 2.061 & 2.912 \\
$\mathbf{8 n}$ & -9.6 & 1.265 & 1.506 \\
$\mathbf{8 0}$ & -8.9 & 2.170 & 2.873 \\
$\mathbf{8 p}$ & -9.1 & 4.238 & 6.490 \\
$\mathbf{8 q}$ & -10.9 & 0.000 & 0.000 \\
$\mathbf{8 r}$ & -10.5 & 0.000 & 0.000 \\
Native ligand & -7.9 & 0.000 & 0.000 \\
Plumbagin & -6.2 & 1.357 & 2.189 \\
& & &
\end{tabular}

value of partition coefficient $\log$ P clearly defines the lipophilic nature of the compounds $\mathbf{8 b}, \mathbf{8 d}, \mathbf{8 e}$ and ability to permeate cell membrane effectively and efficiently.

The molecular docking of the discussed conjugates has highlighted critical pharmacophoric features of the potential drug candidates which directly affect their inhibitory activity against MCF-7 cell-line. It is evident that the synthesized tetrahydro- $\beta$-carboline-isatin conjugates are promising antiproliferative agents. With further derivatization, the pharmacological efficacy of the potential drug candidates can be improved to reveal potent inhibitors. The binding pattern of the active compound and potential anti-proliferative agents i.e. compounds $\mathbf{8 b}, \mathbf{8 d}, \mathbf{8 e}$ and $\mathbf{8 r}$, respectively; emphasises the need for the active ligand to comprise hydrophobic pharmacophores. Effective inhibitory activity can be achieved from the presence of hydrogen bonds created between the protein and ligand, specifically with the conjugate being the hydrogen bond acceptor and protein the donor. Further to this, the interaction of the isatin conjugate in the binding pocket should be facilitated with the presence of ionised non-covalent bonds. The structural incentive by introducing a halogen at position C-5 position of the isatin appears not critical as is observed in compound $\mathbf{8 r}$.

\section{Conclusion}

A series of tetrahydro- $\beta$-carboline-isatin conjugates having varied substituents at C-5 position of isatin as well as at C-1 position of TH $\beta C$ ring were prepared via azide alkyne cycloaddition reactions. The compound, $\mathbf{8 b}$ with an optimum combination of unsubstituted TH $\beta C$ and isatin ring along with a propyl-chain as spacer, proved to be most potent among the synthesized scaffolds exhibiting an $\mathrm{IC}_{50}$ value of $37.42 \mu \mathrm{M}$, comparable to that of peganumine A. The anti-proliferative evaluation studies of synthesized conjugates revealed them to be selective against estrogen responsive MCF-7 cell line suggestive of the fact that they can serve as molecular templates in the expansion of cancer chemotherapies competent of targeting ER-dependent breast cancers. Docking results further support that the most potent compound displays great binding affinity with $\operatorname{ER}(+)$ owing to occupation of binding sites via various hydrophobic, $\mathrm{H}$-bonding and cation- $\pi$ interactions with $\operatorname{ER}(+)$ active site.

\section{Experimental section}

\section{General note}

All the reactions were carried out using standard techniques. The column chromatography was carried out using silica gel (60-120 mesh) with ethyl acetate : hexane as eluent. Melting points were recorded in capillaries and are uncorrected. The spectra of ${ }^{1} \mathrm{H}$ NMR and ${ }^{13} \mathrm{C}$ NMR spectra were recorded on JEOL 400, BRUKER 500 and $125 \mathrm{MHz}$ spectrometers and obtained as $\mathrm{CDCl}_{3}$ solutions using tetramethylsilane (TMS) as an internal standard. Chemical shifts were reported in parts per million (ppm) and coupling constants $J$ indicated in hertz. Mass spectral data was gathered on BrukermicrOTOF QII equipment using ESI as the source.

Preparation of 2-prop-2-ynyl-2,3,4,9-tetrahydro- $\beta$-carboline-3carboxylic acid ethyl ester 4

To a solution of secondary amine $(3 \mathrm{mmol})$ and $\mathrm{K}_{2} \mathrm{CO}_{3}(3.6$ $\mathrm{mmol})$ in $15 \mathrm{~mL}$ acetone, propargyl bromide $(3.6 \mathrm{mmol})$ was added and reaction mixture was stirred overnight at room temperature. The reaction mixture was filtered and concentrated under reduced pressure to yield crude product which was purified via column chromatography using $(20: 80)$ ethylacetate : hexane as eluent. 4a pale yellow solid; $92 \%$; $4 \mathbf{b}$ pale yellow solid $90 \%$; 4 c light brown solid $84 \%$.

\section{Procedure for the synthesis of conjugates $(8 a-r)$}

To the stirred solution of $N$-alkylazido-isatin $7(1 \mathrm{mmol})$ and 2prop-2-ynyl-2,3,4,9-tetrahydro- $\beta$-carboline-3-carboxylic acid ethyl ester 4 in a mixture of ethanol : water $(85: 15)$, was added $\mathrm{CuSO}_{4} \cdot 5 \mathrm{H}_{2} \mathrm{O}(0.055 \mathrm{mmol})$ and sodium ascorbate $(0.143$ $\mathrm{mmol}$ ). The reaction mixture was allowed to stir at $\mathrm{rt}$ for 7-8 $\mathrm{h}$. Upon completion of the reaction as monitored through TLC, the resulting mixture was extracted with ethyl acetate $(2 \times 30 \mathrm{~mL})$ and water $(2 \times 25 \mathrm{~mL})$. The organic layers were combined, dried over anhydrous $\mathrm{Na}_{2} \mathrm{SO}_{4}$ and concentrated under reduced pressure to yield the desired conjugates (8a-r); which were purified via chromatography using alumina as stationary phase and ethyl-acetate : hexane $(80: 20)$ as eluent mixture.

2-\{1-[2-(2,3-Dioxo-2,3-dihydro-indol-1-yl)-ethyl]-1 $H$-[1,2,3]triazol-4-ylmethyl\}-2,3,4,9-tetrahydro- $1 \mathrm{H}$ - $\beta$-carboline-3-carboxylic acid ethyl ester (8a). Yield $72 \%$; light orange solid; mp 108$110{ }^{\circ} \mathrm{C} ;{ }^{1} \mathrm{H}$ NMR $\left(400 \mathrm{MHz}, \mathrm{CDCl}_{3}\right) \delta 1.20(\mathrm{t}, J=7.1 \mathrm{~Hz}, 3 \mathrm{H}$, $\left.-\mathrm{CH}_{3}\right) ; 2.93-3.10\left(\mathrm{~m}, 2 \mathrm{H}, \mathrm{H}^{4}\right) ; 3.58-3.66\left(\mathrm{~m}, 2 \mathrm{H}, \mathrm{H}^{3}+\mathrm{H}^{5}\right) ; 3.99(\mathrm{~s}$, $\left.2 \mathrm{H}, \mathrm{H}^{6}\right) ; 4.03-4.15\left(\mathrm{~m}, 5 \mathrm{H}, \mathrm{H}^{5}+\mathrm{H}^{2}+\mathrm{H}^{8}\right) ; 4.62(\mathrm{t}, J=5.6 \mathrm{~Hz}, 2 \mathrm{H}$, $\left.\mathrm{H}^{7}\right) ; 6.53(\mathrm{~d}, J=8 \mathrm{~Hz}, 1 \mathrm{H}, \mathrm{Ar}-\mathrm{H}) ; 6.88(\mathrm{t}, J=7.5 \mathrm{~Hz}, 1 \mathrm{H}, \mathrm{Ar}-\mathrm{H})$; 7.02-7.10 (m, 2H, Ar-H); $7.26(\mathrm{~d}, 1 \mathrm{H}, J=7.9 \mathrm{~Hz}, \mathrm{Ar}-\mathrm{H}) ; 7.34-$ $7.41(\mathrm{~m}, 2 \mathrm{H}, \mathrm{Ar}-\mathrm{H}) ; 7.46(\mathrm{~d}, J=7.4 \mathrm{~Hz}, 1 \mathrm{H}, \mathrm{Ar}-\mathrm{H}), 7.56(\mathrm{~s}, 1 \mathrm{H}$, 
triazole-H); $8.30\left(\mathrm{~s}, 1 \mathrm{H},-\mathrm{NH}\right.$ (exchangeable with $\left.\left.\mathrm{D}_{2} \mathrm{O}\right)\right){ }^{13} \mathrm{C}$ NMR $\left(100 \mathrm{MHz}, \mathrm{CDCl}_{3}\right) \delta 14.2,24.0,39.6,45.0,47.4,48.9$, 57.6, 60.4, $106.2,110.1,111.2,117.5,117.8,119.5,121.2$, 124.0, 124.2, 125.4, 127.1, 131.2, 132.4, 135.2, 136.1, 146.2, 158.1, 172.2, 183.0 HRMS calcd for $\mathrm{C}_{27} \mathrm{H}_{26} \mathrm{~N}_{6} \mathrm{O}_{4}[\mathrm{M}+1]^{+} 499.2049$, found 499.2021.

2-\{1-[3-(2,3-Dioxo-2,3-dihydro-indol-1-yl)-propyl]-1 $H$-[1,2,3] triazol-4-ylmethyl\}-2,3,4,9-tetrahydro- $1 \mathrm{H}$ - $\beta$-carboline-3-carboxylic acid ethyl ester (8b). Yield 70\%; light orange solid; mp 101$103{ }^{\circ} \mathrm{C} ;{ }^{1} \mathrm{H}$ NMR $\left(400 \mathrm{MHz}, \mathrm{CDCl}_{3}\right) \delta 1.23\left(\mathrm{t}, J=7.1 \mathrm{~Hz}, 3 \mathrm{H},-\mathrm{CH}_{3}\right)$; 2.32-2.39 (m, 2H, $\left.\mathrm{H}^{8}\right)$; 3.09-3.21 (m, $\left.2 \mathrm{H}, \mathrm{H}^{4}\right) ; 3.75(\mathrm{t}, J=6.8 \mathrm{~Hz}$, $\left.2 \mathrm{H}, \mathrm{H}^{9}\right) ; 3.88-3.93\left(\mathrm{~m}, 2 \mathrm{H}, \mathrm{H}^{3}+\mathrm{H}^{5}\right) ; 4.09-4.23\left(\mathrm{~m}, 5 \mathrm{H}, \mathrm{H}^{5}+\mathrm{H}^{2}+\right.$ $\left.\mathrm{H}^{6}\right) ; 4.40\left(\mathrm{t}, J=6.4 \mathrm{~Hz}, 2 \mathrm{H}, \mathrm{H}^{7}\right) ; 6.88(\mathrm{~d}, J=7.9 \mathrm{~Hz}, 1 \mathrm{H}, \mathrm{Ar}-\mathrm{H})$; 7.03-7.12 (m, 3H, Ar-H); 7.28 (d, $J=7.8 \mathrm{~Hz}, 1 \mathrm{H}, \mathrm{Ar}-\mathrm{H}) ; 7.44$ (d, $J$ $=7.4 \mathrm{~Hz}, 1 \mathrm{H}, \mathrm{Ar}-\mathrm{H}) ; 7.53-7.59(\mathrm{~m}, 2 \mathrm{H}, \mathrm{Ar}-\mathrm{H}) ; 7.67(\mathrm{~s}, 1 \mathrm{H}$, triazole$\mathrm{H}) ; 8.03\left(\mathrm{~s}, 1 \mathrm{H},-\mathrm{NH}\right.$ (exchangeable with $\left.\left.\mathrm{D}_{2} \mathrm{O}\right)\right){ }^{13} \mathrm{C}$ NMR $(100 \mathrm{MHz}$, $\left.\mathrm{CDCl}_{3}\right) \delta$ (ppm): 14.3, 23.9, 27.7, 37.5, 46.3, 47.6, 49.2, 60.2, 60.8, 106.1, 110.2, 110.9, 117.6, 117.9, 119.3, 121.5, 123.7, 124.2, 125.8, 127.1, 131.2, 136.2, 138.7, 145.8, 150.3, 158.5, 172.7, 183.0 HRMS calcd for $\mathrm{C}_{28} \mathrm{H}_{28} \mathrm{~N}_{6} \mathrm{O}_{4}[\mathrm{M}+1]^{+}$513.2206, found 513.2234.

2-\{1-[4-(2,3-Dioxo-2,3-dihydro-indol-1-yl)-butyl]-1H-[1,2,3]triazol-4-ylmethyl\}-2,3,4,9-tetrahydro- $1 \mathrm{H}$ - $\beta$-carboline-3-carboxylic acid ethyl ester (8c). Yield $72 \%$; light orange solid; mp $92-94{ }^{\circ} \mathrm{C}$; ${ }^{1} \mathrm{H}$ NMR (400 MHz, $\left.\mathrm{CDCl}_{3}-\mathrm{d}_{1}\right) \delta(\mathrm{ppm}): 1.20(\mathrm{t}, J=7.1 \mathrm{~Hz}, 3 \mathrm{H}$, $\left.-\mathrm{CH}_{3}\right)$; 1.58-1.65 (m, 2H, H $\left.{ }^{9}\right)$; 1.87-1.95 (m, 2H, H $\left.{ }^{8}\right)$; 3.05-3.17 $\left(\mathrm{m}, 2 \mathrm{H}, \mathrm{H}^{4}\right) ; 3.65\left(\mathrm{~m}, 2 \mathrm{H}, \mathrm{H}^{10}\right) ; 3.79\left(\mathrm{~d}, J=15.2 \mathrm{~Hz}, 1 \mathrm{H}, \mathrm{H}^{5}\right) ; 3.86$ $\left(\mathrm{t}, J=5.6 \mathrm{~Hz}, 1 \mathrm{H}, \mathrm{H}^{3}\right) ; 4.00-4.15\left(\mathrm{~m}, 5 \mathrm{H}, \mathrm{H}^{5}+\mathrm{H}^{2}+\mathrm{H}^{6}\right) ; 4.35(\mathrm{t}, J$ $\left.=6.8 \mathrm{~Hz}, 2 \mathrm{H}, \mathrm{H}^{7}\right) ; 6.81(\mathrm{~d}, J=7.9 \mathrm{~Hz}, 1 \mathrm{H}, \mathrm{Ar}-\mathrm{H}) ; 7.00-7.09(\mathrm{~m}$, $3 \mathrm{H}, \mathrm{Ar}-\mathrm{H}) ; 7.27-7.29$ (m, 1H, Ar-H); 7.40 (d, J=7.5 Hz, 1H, Ar$\mathrm{H}) ; 7.48-7.54(\mathrm{~m}, 3 \mathrm{H}, \mathrm{Ar}-\mathrm{H}+$ triazole-H $) ; 8.5(\mathrm{~s}, 1 \mathrm{H},-\mathrm{NH}$ (exchangeable with $\left.\left.\mathrm{D}_{2} \mathrm{O}\right)\right){ }^{13} \mathrm{C} \mathrm{NMR}\left(100 \mathrm{MHz}, \mathrm{CDCl}_{3}\right) \delta(\mathrm{ppm})$ : 14.3, 24.0, 27.4, 29.7, 39.3, 46.2, 49.2, 49.3, 60.3, 60.8, 105.7, $110.1,111.1,117.5,117.8,119.1,121.3,123.1,124.0,125.7$, $127.0,131.4,136.3,138.6,145.6,150.5,158.4,172.82,183.36$ HRMS calcd for $\mathrm{C}_{29} \mathrm{H}_{30} \mathrm{~N}_{6} \mathrm{O}_{4}[\mathrm{M}+1]^{+} 527.2362$, found 527.2324.

2-\{1-[2-(2,3-Dioxo-2,3-dihydro-indol-1-yl)-ethyl]- $1 \mathrm{H}$-[1,2,3]triazol-4-ylmethyl\}-1-methyl-2,3,4,9-tetrahydro-1 $H$ - $\beta$-carboline-3carboxylic acid ethyl ester (8d). Yield 67\%; light orange solid; mp 146-148 ${ }^{\circ} \mathrm{C}^{1} \mathrm{H}$ NMR (400 MHz, $\left.\mathrm{CDCl}_{3}\right) \delta 1.28(\mathrm{t}, J=7.1 \mathrm{~Hz}$, $\left.3 \mathrm{H},-\mathrm{CH}_{3}\right) ; 1.44\left(\mathrm{~d}, J=6.6 \mathrm{~Hz}, 3 \mathrm{H},-\mathrm{CH}_{3}\right) ; 2.70-2.75\left(\mathrm{~m}, 1 \mathrm{H}, \mathrm{H}^{4}\right)$; 2.90-2.94 (m, 1H, H $\left.\mathrm{H}^{4}\right) ; 3.82\left(\mathrm{q}, J=6.4 \mathrm{~Hz}, 1 \mathrm{H}, \mathrm{H}^{5}\right) ; 3.92(\mathrm{~d}, J=$ $\left.15.9 \mathrm{~Hz}, 1 \mathrm{H}, \mathrm{H}^{6}\right)$; 4.16-4.20 (m, 5H, $\left.\mathrm{H}^{6}+\mathrm{H}^{2}+\mathrm{H}^{3}+\mathrm{H}^{8}\right)$; 4.61-4.76 $\left(\mathrm{m}, 3 \mathrm{H}, \mathrm{H}^{7}+\mathrm{H}^{8}\right) ; 6.39(\mathrm{~d}, J=7.0 \mathrm{~Hz}, 1 \mathrm{H}, \mathrm{Ar}-\mathrm{H}) ; 7.00-7.14(\mathrm{~m}$, $4 \mathrm{H}, \mathrm{Ar}-\mathrm{H}) ; 7.30$ (d, $J=7.6 \mathrm{~Hz}, 2 \mathrm{H}, \mathrm{Ar}-\mathrm{H}) ; 7.41(\mathrm{~d}, J=7.4 \mathrm{~Hz}, 1 \mathrm{H}$, $\mathrm{Ar}-\mathrm{H}) ; 7.53$ (s, 1H, triazole-H); 8.07 (s, 1H, Ar-H) ${ }^{13} \mathrm{C}$ NMR (100 $\left.\mathrm{MHz}, \mathrm{CDCl}_{3}\right) \delta 14.3,18.3,24.0,45.3,47.4,49.3,49.5,60.4,60.8$, $105.2,110.2$, 111.4, 117.4, 117.5, 119.6, 121.3, 124.2, 125.2, 125.4, 127.1, 130.2, 136.5, 139.7, 146.0, 151.2, 158.6, 172.9, 183.1 HRMS calcd for $\mathrm{C}_{28} \mathrm{H}_{28} \mathrm{~N}_{6} \mathrm{O}_{4}[\mathrm{M}+1]^{+}$513.2206, found 513.2214.

2-\{1-[3-(2,3-Dioxo-2,3-dihydro-indol-1-yl)-propyl]-1H-[1,2,3] triazol-4-ylmethyl\}-1-methyl-2,3,4,9-tetrahydro- $1 \mathrm{H}$ - $\beta$-carboline3-carboxylic acid ethyl ester (8e). Yield 72\%; light orange solid; mp 141-143 ${ }^{\circ} \mathrm{C},{ }^{1} \mathrm{H}$ NMR (400 MHz, $\left.\mathrm{CDCl}_{3}\right) \delta 1.30(\mathrm{t}, J=7.1 \mathrm{~Hz}$, $\left.3 \mathrm{H},-\mathrm{CH}_{3}\right) ; 1.46\left(\mathrm{~d}, J=6.8 \mathrm{~Hz}, 3 \mathrm{H},-\mathrm{CH}_{3}\right), 2.27-2.34\left(\mathrm{~m}, 2 \mathrm{H}, \mathrm{H}^{8}\right)$; 2.93-2.98 (m, $\left.1 \mathrm{H}, \mathrm{H}^{4}\right) ; 3.08-3.15\left(\mathrm{~m}, 1 \mathrm{H}, \mathrm{H}^{4}\right) ; 3.68-3.73(\mathrm{~m}, 3 \mathrm{H}$, $\left.\mathrm{H}^{9}+\mathrm{H}^{3}\right) ; 4.03\left(\mathrm{~d}, J=15.8 \mathrm{~Hz}, 1 \mathrm{H}, \mathrm{H}^{6}\right) ; 4.12(\mathrm{q}, J=6.4 \mathrm{~Hz}, 1 \mathrm{H}$, $\left.\mathrm{H}^{5}\right) ; 4.22-4.27\left(\mathrm{~m}, 3 \mathrm{H}, \mathrm{H}^{2}+\mathrm{H}^{6}\right) ; 4.37\left(\mathrm{t}, J=6.56 \mathrm{~Hz}, 2 \mathrm{H}, \mathrm{H}^{7}\right) ; 6.81$ $(\mathrm{d}, J=7.9 \mathrm{~Hz}, 1 \mathrm{H}, \mathrm{Ar}-\mathrm{H}) ; 7.01-7.09$ (m, 3H, Ar-H); 7.27 (d, $J=$
$8 \mathrm{~Hz}, 1 \mathrm{H}, \mathrm{Ar}-\mathrm{H}) ; 7.41$ (d, $J=7.4 \mathrm{~Hz}, 1 \mathrm{H}, \mathrm{Ar}-\mathrm{H}) ; 7.46(\mathrm{t}, J=7.7 \mathrm{~Hz}$, $1 \mathrm{H}, \mathrm{Ar}-\mathrm{H}) ; 7.52(\mathrm{~d}, J=7.3 \mathrm{~Hz}, 1 \mathrm{H}, \mathrm{Ar}-\mathrm{H}) ; 7.76(\mathrm{~s}, 1 \mathrm{H}$, triazole-H); $8.36\left(\mathrm{~s}, 1 \mathrm{H},-\mathrm{NH}\left(\right.\right.$ exchangeable with $\left.\left.\mathrm{D}_{2} \mathrm{O}\right)\right){ }^{13} \mathrm{C}$ NMR $(100 \mathrm{MHz}$, $\left.\mathrm{CDCl}_{3}\right) \delta 14.3,18.2,24.1,27.8,36.5,46.4,47.6,49.2$, 60.4, 60.7, $105.8,110.1,110.9,116.5,117.8,119.2$, 121.6, 122.7, 124.2, 125.9, 127.5, 131.1, 136.2, 137.7, 146.8, 150.2, 158.5, 172.8, 183.2 HRMS calcd for $\mathrm{C}_{29} \mathrm{H}_{30} \mathrm{~N}_{6} \mathrm{O}_{4}[\mathrm{M}+1]^{+} 527.2362$, found 527.2329.

2-\{1-[4-(2,3-Dioxo-2,3-dihydro-indol-1-yl)-butyl]-1 H-[1,2,3]triazol-4-ylmethyl\}-1-methyl-2,3,4,9-tetrahydro- $1 \mathrm{H}$ - $\boldsymbol{\beta}$-carboline-3carboxylic acid ethyl ester (8f). Yield 68\%; light orange solid; mp 130-134 ${ }^{\circ} \mathrm{C} ;{ }^{1} \mathrm{H}$ NMR (400 MHz, $\left.\mathrm{CDCl}_{3}-\mathrm{d}_{1}\right) \delta 1.29(\mathrm{t}, J=7.0 \mathrm{~Hz}$, $\left.3 \mathrm{H},-\mathrm{CH}_{3}\right) ; 1.41\left(\mathrm{~d}, J=6.3 \mathrm{~Hz}, 3 \mathrm{H},-\mathrm{CH}_{3}\right) ; 1.59-1.63\left(\mathrm{~m}, 2 \mathrm{H}, \mathrm{H}^{9}\right)$; 1.88-1.95 (m, 2H, $\left.\mathrm{H}^{8}\right)$; 2.91-2.95 (m, $\left.1 \mathrm{H}, \mathrm{H}^{4}\right)$; 3.06-3.12 (m, $1 \mathrm{H}$, $\left.\mathrm{H}^{4}\right)$; 3.61-3.70 (m, 3H, $\left.\mathrm{H}^{10}+\mathrm{H}^{3}\right)$; 3.98-4.02 (d, $J=15.7 \mathrm{~Hz}, 1 \mathrm{H}$, $\left.\mathrm{H}^{6}\right)$; 4.06-4.12 (q, $\left.J=6.4 \mathrm{~Hz}, 1 \mathrm{H}, \mathrm{H}^{5}\right) ; 4.19-4.25\left(\mathrm{~m}, 3 \mathrm{H}, \mathrm{H}^{2}+\mathrm{H}^{6}\right)$; $4.35\left(\mathrm{t}, J=6.2 \mathrm{~Hz}, 2 \mathrm{H}, \mathrm{H}^{8}\right) ; 6.76(\mathrm{~d}, J=7.8 \mathrm{~Hz}, 1 \mathrm{H}, \mathrm{Ar}-\mathrm{H})$; 6.967.07 (m, 3H, Ar-H); 7.28 (d, J= 7.6 Hz, 1H, Ar-H); 7.38-7.44 (m, $2 \mathrm{H}, \mathrm{Ar}-\mathrm{H}) ; 7.48(\mathrm{~d}, J=7.08 \mathrm{~Hz}, 1 \mathrm{H}, \mathrm{Ar}-\mathrm{H}) ; 7.65$ (s, 1H, triazole-H); $8.81\left(\mathrm{~s}, 1 \mathrm{H},-\mathrm{NH}\right.$ (exchangeable with $\left.\left.\mathrm{D}_{2} \mathrm{O}\right)\right) .{ }^{13} \mathrm{C}$ NMR $(100 \mathrm{MHz}$, $\left.\mathrm{CDCl}_{3}\right) \delta 14.3,18.5,24.1,27.4,31.6,39.2,44.9,49.3,52.4,61.2$, 61.7, 105.7, 110.1, 111.1, 117.5, 117.9, 119.3, 121.4, 123.7, 123.9, 125.5, 126.8, 135.4, 136.3, 138.6, 145.1, 150.5, 158.3, 173.5, 183.3 HRMS calcd for $\mathrm{C}_{30} \mathrm{H}_{32} \mathrm{~N}_{6} \mathrm{O}_{4}[\mathrm{M}+1]^{+} 541.2519$, found 541.2530.

2-\{1-[3-(2,3-Dioxo-2,3-dihydro-indol-1-yl)-propyl]-1 H-[1,2,3] triazol-4-ylmethyl\}-1-phenyl-2,3,4,9-tetrahydro- $1 \mathrm{H}$ - $\boldsymbol{\beta}$-carboline3-carboxylic acid ethyl ester (8g). Yield 64\%; light orange solid; mp 168-170 ${ }^{\circ} \mathrm{C} ;{ }^{1} \mathrm{H}$ NMR (400 MHz, $\left.\mathrm{CDCl}_{3}\right) \delta 1.17(\mathrm{t}, J=7.1 \mathrm{~Hz}$, $\left.3 \mathrm{H},-\mathrm{CH}_{3}\right)$; 2.95-3.13 (m, 2H, $\left.\mathrm{H}^{4}\right) ; 3.69-3.72\left(\mathrm{~m}, 2 \mathrm{H}, \mathrm{H}^{8}\right) ; 4.00-$ $4.18\left(\mathrm{~m}, 5 \mathrm{H}, \mathrm{H}^{2}+\mathrm{H}^{6}+\mathrm{H}^{3}\right)$; 4.56-4.61 (m, 2H, $\left.\mathrm{H}^{7}\right) ; 5.41(\mathrm{~s}, 1 \mathrm{H}$, $\left.\mathrm{H}^{5}\right) ; 6.46(\mathrm{~d}, J=8.0 \mathrm{~Hz}, 1 \mathrm{H}, \mathrm{Ar}-\mathrm{H}), 7.02-7.15(\mathrm{~m}, 5 \mathrm{H}, \mathrm{Ar}-\mathrm{H})$; 7.22-7.24 (m, 2H, Ar-H); 7.27 (s, 2H, Ar-H); 7.37-7.47 (m, 3H, $\mathrm{Ar}-\mathrm{H}) ; 7.49$ (s, 1H, triazole-H); $7.53(\mathrm{dd}, J=6.5,0.9 \mathrm{~Hz}, 1 \mathrm{H},-\mathrm{NH}$ (exchangeable with $\left.\mathrm{D}_{2} \mathrm{O}\right)$ ); ${ }^{13} \mathrm{C} \mathrm{NMR}\left(100 \mathrm{MHz}, \mathrm{CDCl}_{3}-\mathrm{d}_{1}\right) \delta 14.3$, 24.7, 40.8, 46.0, 47.8, 56.8, 60.5, 60.9, 106.0, 109.5, 110.9, 117.3, 118.2 , 119.3, 121.7, 123.6, 124.2, 125.1, 125.6, 126.9, 128.1, $128.8,129.7,134.7,136.5,138.7,140.3$, 141.9, 147.5, 150.1, 158.5, 172.8, 182.5 HRMS calcd for $\mathrm{C}_{33} \mathrm{H}_{30} \mathrm{~N}_{6} \mathrm{O}_{4}[\mathrm{M}+1]^{+}$ 575.2362 , found 575.2341 .

2-\{1-[3-(2,3-Dioxo-2,3-dihydro-indol-1-yl)-propyl]-1H-[1,2,3] triazol-4-ylmethyl\}-1-phenyl-2,3,4,9-tetrahydro- $1 \mathrm{H}$ - $\beta$-carboline3-carboxylic acid ethyl ester (8h). Yield 68\%; light orange solid; $\mathrm{mp} 163-165{ }^{\circ} \mathrm{C} ;{ }^{1} \mathrm{H} \mathrm{NMR}\left(400 \mathrm{MHz}, \mathrm{CDCl}_{3}\right) \delta 1.17(\mathrm{t}, J=7.1 \mathrm{~Hz}$, $\left.3 \mathrm{H},-\mathrm{CH}_{3}\right) ; 2.27-2.33\left(\mathrm{~m}, 2 \mathrm{H}, \mathrm{H}^{8}\right) ; 3.20-3.22\left(\mathrm{~m}, 2 \mathrm{H}, \mathrm{H}^{4}\right) ; 3.70-$ $3.75\left(\mathrm{~m}, 2 \mathrm{H}, \mathrm{H}^{9}\right) ; 3.85\left(\mathrm{~d}, J=14.8 \mathrm{~Hz}, 1 \mathrm{H}, \mathrm{H}^{6}\right)$; 4.00-4.07 (m, 3H, $\left.\mathrm{H}^{2}+\mathrm{H}^{3}\right) ; 4.14\left(\mathrm{~d}, J=14.7 \mathrm{~Hz}, 1 \mathrm{H}, \mathrm{H}^{6}\right) ; 4.30-4.34\left(\mathrm{~m}, 2 \mathrm{H}_{1} \mathrm{H}^{7}\right)$; $5.51\left(\mathrm{~s}, 1 \mathrm{H}, \mathrm{H}^{5}\right) ; 6.85(\mathrm{~d}, J=7.9 \mathrm{~Hz}, 1 \mathrm{H}, \mathrm{Ar}-\mathrm{H}) ; 7.02-7.16(\mathrm{~m}, 5 \mathrm{H}$, $\mathrm{Ar}-\mathrm{H})$; 7.26-7.29 (m, 2H, Ar-H); 7.43 (s, 1H, Ar-H); 7.46-7.58 (m, $4 \mathrm{H}, \quad \mathrm{Ar}-\mathrm{H}) ; 7.64(\mathrm{~s}, \quad 1 \mathrm{H}$, triazole-H); $7.95(\mathrm{~s}, 1 \mathrm{H},-\mathrm{NH}$ (exchangeable with $\left.\left.\mathrm{D}_{2} \mathrm{O}\right)\right){ }^{13} \mathrm{C}$ NMR $\left(100 \mathrm{MHz}, \mathrm{CDCl}_{3}\right) \delta 14.2$, 24.7, 31.5, 37.5, 46.2 , 47.5, 57.1, 60.5, 61.0, 106.1, 110.2, 110.9, $117.6,118.2$, 119.3, 121.6, 123.2, 124.1, 125.7, 127.0, 128.1, $128.8,128.9,134.8,136.6,138.7,140.6$, 142.1, 147.0, 150.3, 158.5, 173.1, 183.1 HRMS calcd for $\mathrm{C}_{34} \mathrm{H}_{32} \mathrm{~N}_{6} \mathrm{O}_{4}[\mathrm{M}+1]^{+}$ 589.2519 , found 589.2542 .

2-\{1-[4-(2,3-Dioxo-2,3-dihydro-indol-1-yl)-butyl]-1H-[1,2,3] triazol-4-ylmethyl\}-1-phenyl-2,3,4,9-tetrahydro-1H- $\beta$-carboline3-carboxylic acid ethyl ester (8i). Yield 62\%; light orange solid; 
$\operatorname{mp} 158-161{ }^{\circ} \mathrm{C} ;{ }^{1} \mathrm{H}$ NMR $\left(400 \mathrm{MHz}, \mathrm{CDCl}_{3}\right) \delta 1.17(\mathrm{t}, J=7.1 \mathrm{~Hz}$, $\left.3 \mathrm{H},-\mathrm{CH}_{3}\right) ; 1.62-1.69\left(\mathrm{~m}, 2 \mathrm{H}, \mathrm{H}^{9}\right) ; 1.88-1.94\left(\mathrm{~m}, 2 \mathrm{H}, \mathrm{H}^{8}\right) ; 3.13-$ $3.24\left(\mathrm{~m}, 2 \mathrm{H}, \mathrm{H}^{4}\right) ; 3.67-3.71\left(\mathrm{~m}, 2 \mathrm{H}, \mathrm{H}^{10}\right) ; 3.82(\mathrm{~d}, J=14.7 \mathrm{~Hz}, 1 \mathrm{H}$, $\left.\mathrm{H}^{6}\right)$; 3.98-4.07 (m, 3H, $\left.\mathrm{H}^{2}+\mathrm{H}^{3}\right)$; $4.12\left(\mathrm{~d}, J=14.6 \mathrm{~Hz}, 1 \mathrm{H}, \mathrm{H}^{6}\right)$; 4.32-4.36 (m, 2H, H $\left.\mathrm{H}^{7}\right) ; 5.49\left(\mathrm{~s}, 1 \mathrm{H}, \mathrm{H}^{5}\right) ; 6.83(\mathrm{~d}, J=7.8 \mathrm{~Hz}, 1 \mathrm{H}$, $\mathrm{Ar}-\mathrm{H}$ ); 7.04-7.17 (m, 5H, Ar-H); 7.27 (s, 2H, Ar-H); 7.34-7.36 (m, $2 \mathrm{H}, \mathrm{Ar}-\mathrm{H}) ; 7.46-7.53$ (m, 3H, Ar-H); 7.55 (s, 1H, triazole-H); 7.57 $\left(\mathrm{s}, 1 \mathrm{H},-\mathrm{NH}\right.$ (exchangeable with $\left.\mathrm{D}_{2} \mathrm{O}\right)$ ) ${ }^{13} \mathrm{C}$ NMR $(100 \mathrm{MHz}$, $\left.\mathrm{CDCl}_{3}\right) \delta 14.3,24.1,24.6,27.5,39.2,46.1,49.3$, 57.0, 60.6, 60.9, 106.2 , 110.2, 111.0, 117.6, 118.2, 119.3, 121.6, 122.4, 124.0, $125.7,126.9,128.2,128.8,128.9$, 129.7, 134.7, 136.5, 138.6, 142.0, 147.1, 150.5, 158.4, 173.1, 183.3 HRMS calcd for $\mathrm{C}_{35} \mathrm{H}_{34} \mathrm{~N}_{6} \mathrm{O}_{4}[\mathrm{M}+1]^{+}$603.2675, found 603.2681.

2-\{1-[2-(5-Fluoro-2,3-dioxo-2,3-dihydro-indol-1-yl)-ethyl]-1H$[1,2,3]$ triazol-4-ylmethyl $\}-2,3,4,9$-tetrahydro- $1 H$ - $\beta$-carboline-3carboxylic acid ethyl ester (8j). Yield 78\%; light brown solid; mp 134-135 ${ }^{\circ} \mathrm{C} ;{ }^{1} \mathrm{H}$ NMR (500 MHz, $\left.\mathrm{CDCl}_{3}\right) \delta 1.24(\mathrm{t}, J=7.1 \mathrm{~Hz}, 3 \mathrm{H}$, $\left.-\mathrm{CH}_{3}\right) ; 3.00-3.14\left(\mathrm{~m}, 2 \mathrm{H}, \mathrm{H}^{4}\right) ; 3.70-3.73\left(\mathrm{~m}, 2 \mathrm{H}, \mathrm{H}^{3}+\mathrm{H}^{5}\right) ; 4.03(\mathrm{~s}$, $\left.2 \mathrm{H}, \mathrm{H}^{6}\right)$; 4.06-4.19 (m, 5H, $\left.\mathrm{H}^{5}+\mathrm{H}^{2}+\mathrm{H}^{8}\right)$; 4.65 (m, 2H, $\left.\mathrm{H}^{7}\right)$; 6.55$6.58(\mathrm{~m}, 1 \mathrm{H}, \mathrm{Ar}-\mathrm{H}) ; 7.06-7.22(\mathrm{~m}, 4 \mathrm{H}, \mathrm{Ar}-\mathrm{H}) ; 7.29$ (d, J=6.8 Hz, $1 \mathrm{H}, \mathrm{Ar}-\mathrm{H}) ; 7.43(\mathrm{~d}, J=7.7 \mathrm{~Hz}, 1 \mathrm{H}, \mathrm{Ar}-\mathrm{H}) ; 7.61$ (s, $1 \mathrm{H}$, triazole-H); $8.24\left(\mathrm{~s}, 1 \mathrm{H},-\mathrm{NH}\right.$ (exchangeable with $\left.\left.\mathrm{D}_{2} \mathrm{O}\right)\right)^{13} \mathrm{C}$ NMR $(125 \mathrm{MHz}$, $\mathrm{CDCl}_{3}$ ) 14.2, 24.0, 40.7, 46.0, 47.5, 49.0, 59.8, 60.7, 105.8, 110.8, $112.5,112.7,117.7,117.9,119.2$, 121.4, 124.2, 124.8, 125.0, 126.9, 131.1, 136.18, 146.03, 158.2, 172.5, 181.85 HRMS calcd for $\mathrm{C}_{27} \mathrm{H}_{25} \mathrm{FN}_{6} \mathrm{O}_{4}[\mathrm{M}+1]^{+} 517.1955$, found 517.1919 .

2-\{1-[3-(5-Fluoro-2,3-dioxo-2,3-dihydro-indol-1-yl)-propyl]$1 H$ - $[1,2,3]$ triazol-4-ylmethyl $\}-2,3,4,9$-tetrahydro- $1 H$ - $\beta$-carboline3-carboxylic acid ethyl ester (8k). Yield 74\%; light brown solid; mp 128-130 ${ }^{\circ} \mathrm{C} ;{ }^{1} \mathrm{H}$ NMR (400 MHz, $\left.\mathrm{CDCl}_{3}\right) \delta 1.2(\mathrm{t}, J=7.1 \mathrm{~Hz}$, $\left.3 \mathrm{H},-\mathrm{CH}_{3}\right) ; 2.39-2.43\left(\mathrm{~m}, 2 \mathrm{H}, \mathrm{H}^{8}\right) ; 3.18-3.32\left(\mathrm{~m}, 2 \mathrm{H}, \mathrm{H}^{4}\right) ; 3.96(\mathrm{t}$, $\left.J=6.8 \mathrm{~Hz}, 2 \mathrm{H}, \mathrm{H}^{9}\right) ; 3.99-4.03\left(\mathrm{~m}, 2 \mathrm{H}, \mathrm{H}^{3}+\mathrm{H}^{5}\right) ; 4.12-4.34(\mathrm{~m}$, $\left.5 \mathrm{H}, \mathrm{H}^{5}+\mathrm{H}^{2}+\mathrm{H}^{6}\right) ; 4.45\left(\mathrm{t}, J=6.4 \mathrm{~Hz}, 2 \mathrm{H}, \mathrm{H}^{7}\right) ; 6.98-7.01(\mathrm{~m}, 1 \mathrm{H}$, $\mathrm{Ar}-\mathrm{H})$; 7.05-7.18 (m, 3H, Ar-H); 7.30 (d, $J=7.8 \mathrm{~Hz}, 1 \mathrm{H}, \mathrm{Ar}-\mathrm{H})$; 7.48 (d, $J=7.4 \mathrm{~Hz}, 1 \mathrm{H}, \mathrm{Ar}-\mathrm{H}) ; 7.23-7.58(\mathrm{~m}, 1 \mathrm{H}, \mathrm{Ar}-\mathrm{H}) ; 7.71(\mathrm{~s}$, $1 \mathrm{H}$, triazole-H); $8.05\left(\mathrm{~s}, 1 \mathrm{H},-\mathrm{NH}\right.$ (exchangeable with $\left.\left.\mathrm{D}_{2} \mathrm{O}\right)\right){ }^{13} \mathrm{C}$ NMR (100 MHz, $\left.\mathrm{CDCl}_{3}\right) \delta(\mathrm{ppm}): 14.3,24.0,27.9,37.8,47.3,47.8$, 50.2 , 60.5, 61.2, 105.8, 110.1, 110.3, 116.5, 117.5, 120.1, 121.8, $124.6,125.4,126.3,127.9$, 131.1, 136.7, 138.9, 145.9, 150.2, 158.7, 172.4, 182.4 HRMS calcd for $\mathrm{C}_{28} \mathrm{H}_{27} \mathrm{FN}_{6} \mathrm{O}_{4}[\mathrm{M}+1]^{+}$ 531.2111 , found 531.2132 .

2-\{1-[4-(5-Fluoro-2,3-dioxo-2,3-dihydro-indol-1-yl)-butyl]$1 H$ - $[1,2,3]$ triazol-4-ylmethyl $\}-2,3,4,9$-tetrahydro- $1 H$ - $\beta$-carboline3-carboxylic acid ethyl ester (81). Yield 76\%; light brown solid; mp 121-123 ${ }^{\circ} \mathrm{C} ;{ }^{1} \mathrm{H}$ NMR (400 MHz, $\left.\mathrm{CDCl}_{3}\right) \delta 1.20(\mathrm{t}, J=7.1 \mathrm{~Hz}$, $\left.3 \mathrm{H},-\mathrm{CH}_{3}\right) ; 1.60-1.67\left(\mathrm{~m}, 2 \mathrm{H}, \mathrm{H}^{9}\right) ; 1.90-1.98\left(\mathrm{~m}, 2 \mathrm{H}, \mathrm{H}^{8}\right) ; 3.18-$ $3.33\left(\mathrm{~m}, 2 \mathrm{H}, \mathrm{H}^{4}\right) ; 3.86\left(\mathrm{~m}, 2 \mathrm{H}, \mathrm{H}^{10}\right) ; 3.95\left(\mathrm{~d}, J=15.2 \mathrm{~Hz}, 1 \mathrm{H}, \mathrm{H}^{5}\right)$; $4.03\left(\mathrm{t}, J=5.6 \mathrm{~Hz}, 1 \mathrm{H}, \mathrm{H}^{3}\right) ; 4.10-4.33\left(\mathrm{~m}, 5 \mathrm{H}, \mathrm{H}^{5}+\mathrm{H}^{2}+\mathrm{H}^{6}\right) ; 4.46$ $\left(\mathrm{t}, 2 \mathrm{H}, J=6.8 \mathrm{~Hz}, \mathrm{H}^{7}\right) ; 6.83$ (d, $\left.J=7.9 \mathrm{~Hz}, 1 \mathrm{H}, \mathrm{Ar}-\mathrm{H}\right) ; 7.06-7.15$ $(\mathrm{m}, 3 \mathrm{H}, \mathrm{Ar}-\mathrm{H}) ; 7.29-7.32(\mathrm{~m}, 1 \mathrm{H}, \mathrm{Ar}-\mathrm{H}) ; 7.46(\mathrm{~d}, J=7.5 \mathrm{~Hz}, 1 \mathrm{H}$, $\mathrm{Ar}-\mathrm{H}) ; 7.48-7.54$ (m, 2H, Ar-H + triazole-H); $8.43(\mathrm{~s}, 1 \mathrm{H},-\mathrm{NH}$ (exchangeable with $\left.\left.\mathrm{D}_{2} \mathrm{O}\right)\right){ }^{13} \mathrm{C} \mathrm{NMR}\left(100 \mathrm{MHz}, \mathrm{CDCl}_{3}\right) \delta 14.3$, 24.6, 27.7, 30.1, 39.5, 47.2, 49.4, 49.8, 60.6, 60.9, 105.8, 110.3, $111.2,117.9,118.8,119.4,121.7,123.2,124.9$, 125.6, 127.7, 131.2, 136.2 , 140.1, 145.4, 150.8, 158.8, 172.8, 182.3 HRMS calcd for $\mathrm{C}_{29} \mathrm{H}_{29} \mathrm{FN}_{6} \mathrm{O}_{4}[\mathrm{M}+1]^{+}$545.2268, found 545.2291.
2-\{1-[2-(5-Fluoro-2,3-dioxo-2,3-dihydro-indol-1-yl)-ethyl]$1 H$ - $[1,2,3]$ triazol-4-ylmethyl $\}-1-m e t h y l-2,3,4,9-$ tetrahydro- $1 H-\beta$ carboline-3-carboxylic acid ethyl ester (8m). Yield 64\%; light brown solid; mp 118-120 ${ }^{\circ} \mathrm{C} ;{ }^{1} \mathrm{H}$ NMR (400 MHz, $\mathrm{CDCl}_{3}-$ ) $\delta(\mathrm{ppm}): 1.30\left(\mathrm{t}, J=7.1 \mathrm{~Hz}, 3 \mathrm{H},-\mathrm{CH}_{3}\right) ; 1.43(\mathrm{~d}, J=6.6 \mathrm{~Hz}, 3 \mathrm{H}$, $\left.-\mathrm{CH}_{3}\right)$; 2.74-2.79 (m, 1H, $\left.\mathrm{H}^{4}\right)$; 2.95-2.99 (m, 1H, $\left.\mathrm{H}^{4}\right) ; 3.87(\mathrm{q}, J=$ $\left.6.4 \mathrm{~Hz}, 1 \mathrm{H}, \mathrm{H}^{5}\right) ; 3.95\left(\mathrm{~d}, J=15.9 \mathrm{~Hz}, 1 \mathrm{H}, \mathrm{H}^{6}\right) ; 4.20-4.24(\mathrm{~m}, 5 \mathrm{H}$, $\left.\mathrm{H}^{6}+\mathrm{H}^{2}+\mathrm{H}^{3}+\mathrm{H}^{8}\right) ; 4.67-4.81\left(\mathrm{~m}, 3 \mathrm{H}, \mathrm{H}^{7}+\mathrm{H}^{8}\right) ; 6.43(\mathrm{~d}, J=$ $7.0 \mathrm{~Hz}, 1 \mathrm{H}, \mathrm{Ar}-\mathrm{H})$; 7.06-7.17 (m, 3H, Ar-H); 7.37 (d, $J=7.6 \mathrm{~Hz}$, $2 \mathrm{H}, \mathrm{Ar}-\mathrm{H}) ; 7.47$ (d, $J=7.4 \mathrm{~Hz}, 1 \mathrm{H}, \mathrm{Ar}-\mathrm{H}) ; 7.56$ (s, $1 \mathrm{H}$, triazole-H); $8.04(\mathrm{~s}, 1 \mathrm{H}, \mathrm{Ar}-\mathrm{H}){ }^{13} \mathrm{C} \mathrm{NMR}\left(100 \mathrm{MHz}, \mathrm{CDCl}_{3}\right) \delta$ (ppm): 14.3, 18.5, 24.6, 45.8, 47.4, 49.4, 49.7, 60.5, 60.9, 105.1, 110.3, 110.8, $116.4,117.7,120.4,122.4,124.6,125.1,125.7,127.1,131.2$, 135.4, 139.6, 145.4, 151.3, 157.6, 172.8, 182.5 HRMS calcd for $\mathrm{C}_{28} \mathrm{H}_{27} \mathrm{FN}_{6} \mathrm{O}_{4}[\mathrm{M}+1]^{+}$531.2111, found 531.2134.

2-\{1-[3-(5-Fluoro-2,3-dioxo-2,3-dihydro-indol-1-yl)-propyl]$1 H$ - $[1,2,3]$ triazol-4-ylmethyl\}-1-methyl-2,3,4,9-tetrahydro-1H- $\beta$ carboline-3-carboxylic acid ethyl ester (8n). Yield 65\%; light brown solid; mp 112-114 ${ }^{\circ} \mathrm{C} ;{ }^{1} \mathrm{H}$ NMR (400 MHz, $\left.\mathrm{CDCl}_{3}\right) \delta 1.32$ $\left(\mathrm{t}, J=7.1 \mathrm{~Hz}, 3 \mathrm{H},-\mathrm{CH}_{3}\right) ; 1.49\left(\mathrm{~d}, J=6.8 \mathrm{~Hz}, 3 \mathrm{H},-\mathrm{CH}_{3}\right), 2.30-2.37$ $\left(\mathrm{m}, J=15.8 \mathrm{~Hz}, 2 \mathrm{H}, \mathrm{H}^{8}\right) ; 2.98-3.02\left(\mathrm{~m}, 1 \mathrm{H}, \mathrm{H}^{4}\right) ; 3.06-3.18(\mathrm{~m}$, $\left.1 \mathrm{H}, \mathrm{H}^{4}\right) ; 3.70-3.77\left(\mathrm{~m}, 3 \mathrm{H}, \mathrm{H}^{9}+\mathrm{H}^{3}\right) ; 4.06\left(\mathrm{~d}, J=15.8 \mathrm{~Hz}, 1 \mathrm{H}, \mathrm{H}^{6}\right)$; $4.15\left(\mathrm{q}, J=6.4 \mathrm{~Hz}, 1 \mathrm{H}, \mathrm{H}^{5}\right) ; 4.25-4.30\left(\mathrm{~m}, 3 \mathrm{H}, \mathrm{H}^{2}+\mathrm{H}^{6}\right) ; 4.38(\mathrm{t}, J$ $\left.=6.56 \mathrm{~Hz}, 2 \mathrm{H}, \mathrm{H}^{7}\right) ; 6.82(\mathrm{~d}, J=7.9 \mathrm{~Hz}, 1 \mathrm{H}, \mathrm{Ar}-\mathrm{H}) ; 7.13-7.22(\mathrm{~m}$, $2 \mathrm{H}, \mathrm{Ar}-\mathrm{H}) ; 7.30$ (d, $J=8 \mathrm{~Hz}, 1 \mathrm{H}, \mathrm{Ar}-\mathrm{H}) ; 7.48$ (d, $J=7.4 \mathrm{~Hz}, 1 \mathrm{H}$, $\mathrm{Ar}-\mathrm{H})$; 7.52 (t, $J=7.7 \mathrm{~Hz}, 1 \mathrm{H}, \mathrm{Ar}-\mathrm{H}) ; 7.56$ (d, $J=7.3 \mathrm{~Hz}, 1 \mathrm{H}, \mathrm{Ar}-$ $\mathrm{H}) ; 7.78(\mathrm{~s}, 1 \mathrm{H}$, triazole-H); $8.32(\mathrm{~s}, 1 \mathrm{H},-\mathrm{NH}$ (exchangeable with $\left.\left.\mathrm{D}_{2} \mathrm{O}\right)\right){ }^{13} \mathrm{C} \mathrm{NMR}\left(100 \mathrm{MHz}, \mathrm{CDCl}_{3}\right) \delta 14.3,18.5,24.6,27.4,35.5$, 46.7 , 47.8, 49.3, 60.1, 60.6, 105.4, 110.3, 111.4, 116.8, 117.5, 118.2 , 120.8, 122.9, 124.3, 125.8, 127.6, 131.0, 136.8, 137.8, 146.4, 150.1, 158.6, 172.7, 182.8 HRMS calcd for $\mathrm{C}_{29} \mathrm{H}_{29} \mathrm{FN}_{6} \mathrm{O}_{4}$ $[\mathrm{M}+1]^{+} 545.2268$, found 545.2282.

2-\{1-[4-(5-Fluoro-2,3-dioxo-2,3-dihydro-indol-1-yl)-butyl]$1 H$ - $[1,2,3]$ triazol-4-ylmethyl $\}-1-m e t h y l-2,3,4,9$-tetrahydro- $1 H$ - $\beta$ carboline-3-carboxylic acid ethyl ester (80). Yield 67\%; light brown solid; mp 101-103 ${ }^{\circ} \mathrm{C} ;{ }^{1} \mathrm{H}$ NMR (400 $\left.\mathrm{MHz} \mathrm{CDCl}_{3}\right) \delta 1.31$ $\left(\mathrm{t}, J=7.0 \mathrm{~Hz}, 3 \mathrm{H},-\mathrm{CH}_{3}\right) ; 1.42\left(\mathrm{~d}, J=6.3 \mathrm{~Hz}, 3 \mathrm{H},-\mathrm{CH}_{3}\right) ; 1.60-1.67$ $\left(\mathrm{m}, 2 \mathrm{H}, \mathrm{H}^{9}\right) ; 1.90-1.94\left(\mathrm{~m}, 2 \mathrm{H}, \mathrm{H}^{8}\right) ; 2.93-2.98\left(\mathrm{~m}, 1 \mathrm{H}, \mathrm{H}^{4}\right) ; 3.07-$ $3.15\left(\mathrm{~m}, 1 \mathrm{H}, \mathrm{H}^{4}\right) ; 3.68-3.74\left(\mathrm{~m}, 3 \mathrm{H}, \mathrm{H}^{10}+\mathrm{H}^{3}\right)$; 4.01-4.06 (d, $J=$ $\left.15.7 \mathrm{~Hz}, 1 \mathrm{H}, \mathrm{H}^{6}\right) ; 4.08-4.14\left(\mathrm{q}, J=6.4 \mathrm{~Hz}, 1 \mathrm{H}, \mathrm{H}^{5}\right) ; 4.21-4.27(\mathrm{~m}$, $\left.3 \mathrm{H}, \mathrm{H}^{2}+\mathrm{H}^{6}\right) ; 4.39\left(\mathrm{t}, J=6.2 \mathrm{~Hz}, 2 \mathrm{H}, \mathrm{H}^{8}\right) ; 6.78(\mathrm{~d}, J=7.8 \mathrm{~Hz}, 1 \mathrm{H}$, $\mathrm{Ar}-\mathrm{H})$; 6.98-7.11 (m, 2H, Ar-H); 7.30 (d, $J=7.6 \mathrm{~Hz}, 1 \mathrm{H}, \mathrm{Ar}-\mathrm{H})$; 7.34-7.45 (m, 2H, Ar-H); 7.49 (d, J=7.0 Hz, 1H, Ar-H); 7.68 (s, $1 \mathrm{H}$, triazole- $\mathrm{H}) ; 8.87\left(\mathrm{~s}, 1 \mathrm{H},-\mathrm{NH}\right.$ (exchangeable with $\left.\left.\mathrm{D}_{2} \mathrm{O}\right)\right) .{ }^{13} \mathrm{C}$ NMR (100 MHz, $\mathrm{CDCl}_{3}-\mathrm{d}_{1}$ ) $\delta$ (ppm): 14.3, 18.7, 24.5, 27.8, 31.6, 39.2, 45.0, 49.2 , 52.2 , 61.2, 61.8, 105.9, 110.1, 113.1, 117.5, 118.9, $119.8,121.4,122.7,123.9,126.1,126.8,135.4,136.3,138.6$, 146.0, 150.4, 158.2, 173.5, 183.0 HRMS calcd for $\mathrm{C}_{30} \mathrm{H}_{31} \mathrm{FN}_{6} \mathrm{O}_{4}$ $[\mathrm{M}+1]^{+} 559.2424$, found 559.2446.

2-\{1-[2-(5-Fluoro-2,3-dioxo-2,3-dihydro-indol-1-yl)-ethyl]$1 H$ - $[1,2,3]$ triazol-4-ylmethyl\}-1-phenyl-2,3,4,9-tetrahydro-1H- $\beta$ carboline-3-carboxylic acid ethyl ester (8p). Yield 62\%; light brown solid; mp 198-200 ${ }^{\circ} \mathrm{C} ;{ }^{1} \mathrm{H}$ NMR (400 MHz, $\left.\mathrm{CDCl}_{3}\right) \delta 1.18$ $\left(\mathrm{t}, J=7.1 \mathrm{~Hz}, 3 \mathrm{H},-\mathrm{CH}_{3}\right) ; 3.01-3.14\left(\mathrm{~m}, 2 \mathrm{H}, \mathrm{H}^{4}\right) ; 3.70-3.75(\mathrm{~m}$, $\left.2 \mathrm{H}, \mathrm{H}^{8}\right) ; 4.03-4.21\left(\mathrm{~m}, 5 \mathrm{H}, \mathrm{H}^{2}+\mathrm{H}^{6}+\mathrm{H}^{3}\right)$; 4.60-4.67 (m, $\left.2 \mathrm{H}, \mathrm{H}^{7}\right)$; $5.47\left(\mathrm{~s}, 1 \mathrm{H}, \mathrm{H}^{5}\right) ; 6.48(\mathrm{~d}, J=8.0 \mathrm{~Hz}, 1 \mathrm{H}, \mathrm{Ar}-\mathrm{H}), 7.05-7.21(\mathrm{~m}, 4 \mathrm{H}$, 
$\mathrm{Ar}-\mathrm{H})$; 7.24-7.29 (m, 2H, Ar-H); 7.31 (s, 2H, Ar-H); 7.38-7.49 (m, $3 \mathrm{H}, \quad \mathrm{Ar}-\mathrm{H}) ; 7.51(\mathrm{~s}, \quad 1 \mathrm{H}$, triazole-H); $7.54(\mathrm{~s}, \quad 1 \mathrm{H},-\mathrm{NH}$ (exchangeable with $\left.\mathrm{D}_{2} \mathrm{O}\right)$ ); ${ }^{13} \mathrm{C}$ NMR $\left(100 \mathrm{MHz} \mathrm{CDCl}_{3}\right) \delta 14.3$, 24.8, 40.7, 46.2, 47.9, 56.7, 60.5, 61.0, 106.0, 109.4, 110.9, 112.3, $118.2,119.4,121.7,123.6,124.2$, 125.1, 125.8, 126.9, 128.2, $128.9,129.6,134.6,136.5,138.4,140.3,141.8,147.5,150.6$, 158.7, 172.3, 182.8 HRMS calcd for $\mathrm{C}_{33} \mathrm{H}_{29} \mathrm{FN}_{6} \mathrm{O}_{4}[\mathrm{M}+1]^{+}$ 593.2268 , found 593.2287 .

2-\{1-[3-(5-Fluoro-2,3-dioxo-2,3-dihydro-indol-1-yl)-propyl]$1 H$ - $[1,2,3]$ triazol-4-ylmethyl $\}-1-p h e n y l-2,3,4,9$-tetrahydro- $1 H$ - $\beta$ carboline-3-carboxylic acid ethyl ester(8q). Yield 64\%; light brown solid; mp 191-193 ${ }^{\circ} \mathrm{C} ;{ }^{1} \mathrm{H}$ NMR (400 $\left.\mathrm{MHz} \mathrm{CDCl}_{3}\right) \delta 1.17(\mathrm{t}$, $\left.J=7.1 \mathrm{~Hz}, 3 \mathrm{H},-\mathrm{CH}_{3}\right) ; 2.29-2.34\left(\mathrm{~m}, 2 \mathrm{H}, \mathrm{H}^{8}\right) ; 3.23-3.25(\mathrm{~m}, 2 \mathrm{H}$, $\left.\mathrm{H}^{4}\right) ; 3.72-3.78\left(\mathrm{~m}, 2 \mathrm{H}, \mathrm{H}^{9}\right) ; 3.89\left(\mathrm{~d}, J=14.8 \mathrm{~Hz}, 1 \mathrm{H}, \mathrm{H}^{6}\right) ; 4.01-4.08$ $\left(\mathrm{m}, 3 \mathrm{H}, \mathrm{H}^{2}+\mathrm{H}^{3}\right) ; 4.20\left(\mathrm{~d}, J=14.7 \mathrm{~Hz}, 1 \mathrm{H}, \mathrm{H}^{6}\right) ; 4.34-4.37(\mathrm{~m}, 2 \mathrm{H}$, $\left.\mathrm{H}^{7}\right) ; 5.54\left(\mathrm{~s}, 1 \mathrm{H}, \mathrm{H}^{5}\right) ; 6.87(\mathrm{~d}, J=7.9 \mathrm{~Hz}, 1 \mathrm{H}, \mathrm{Ar}-\mathrm{H}) ; 7.03-7.18(\mathrm{~m}$, $4 \mathrm{H}, \mathrm{Ar}-\mathrm{H})$; 7.27-7.30 (m, 2H, Ar-H); 7.42 (s, 1H, Ar-H); 7.47-7.60 $(\mathrm{m}, 4 \mathrm{H}, \mathrm{Ar}-\mathrm{H}) ; 7.68(\mathrm{~s}, 1 \mathrm{H}$, triazole-H) $7.97(\mathrm{~s}, 1 \mathrm{H},-\mathrm{NH}$ (exchangeable with $\left.\mathrm{D}_{2} \mathrm{O}\right){ }^{13} \mathrm{C}$ NMR $\left(100 \mathrm{MHz}, \mathrm{CDCl}_{3}\right) \delta 14.3,24.8$, 31.6, 37.8, 46.4, 47.4, 57.5, 60.4, 61.2, 106.4, 110.3, 110.8, 117.6, 118.5, 119.5, 121.6, 123.1, 124.2, 125.7, 127.1, 128.2, 128.4, 129.1, 134.7, 136.9, 138.9, 140.5, 142.7, 147.1, 150.2, 158.6, 173.1, 183.2 HRMS calcd for $\mathrm{C}_{34} \mathrm{H}_{31} \mathrm{FN}_{6} \mathrm{O}_{4}[\mathrm{M}+1]^{+}$607.2424, found 607.2467.

2-\{1-[4-(5-Fluoro-2,3-dioxo-2,3-dihydro-indol-1-yl)-butyl]$1 H$ - $[1,2,3]$ triazol-4-ylmethyl $\}-1-p h e n y l-2,3,4,9-t e t r a h y d r o-1 H-\beta$ carboline-3-carboxylic acid ethyl ester (8r). Yield 68\%; light brown solid; mp 188-190 ${ }^{\circ} \mathrm{C} ;{ }^{1} \mathrm{H}$ NMR (400 MHz, $\left.\mathrm{CDCl}_{3}\right) \delta 1.17$ $\left(\mathrm{t}, J=7.1 \mathrm{~Hz}, 3 \mathrm{H},-\mathrm{CH}_{3}\right) ; 1.64-1.73\left(\mathrm{~m}, 2 \mathrm{H}, \mathrm{H}^{9}\right) ; 1.92-1.97(\mathrm{~m}$, $\left.2 \mathrm{H}, \mathrm{H}^{8}\right) ; 3.19-3.27\left(\mathrm{~m}, 2 \mathrm{H}, \mathrm{H}^{4}\right) ; 3.68-3.72\left(\mathrm{~m}, 2 \mathrm{H}, \mathrm{H}^{10}\right) ; 3.85(\mathrm{~d}, J$ $\left.=14.7 \mathrm{~Hz}, 1 \mathrm{H}, \mathrm{H}^{6}\right) ; 4.02-4.10\left(\mathrm{~m}, 3 \mathrm{H}, \mathrm{H}^{2}+\mathrm{H}^{3}\right) ; 4.15(\mathrm{~d}, J=$ $\left.14.6 \mathrm{~Hz}, 1 \mathrm{H}, \mathrm{H}^{6}\right) ; 4.35-4.38\left(\mathrm{~m}, 2 \mathrm{H}, \mathrm{H}^{7}\right) ; 5.51\left(\mathrm{~s}, 1 \mathrm{H}, \mathrm{H}^{5}\right) ; 6.89(\mathrm{~d}$, $J=7.8 \mathrm{~Hz}, 1 \mathrm{H}, \mathrm{Ar}-\mathrm{H})$; 7.09-7.21 (m, 4H, Ar-H); 7.29 (s, 2H, Ar$\mathrm{H})$; 7.41-7.46 (m, 2H, Ar-H); 7.48-7.52 (m, 3H, Ar-H); 7.56 (s, $1 \mathrm{H}$, triazole-H); $7.59\left(\mathrm{~s}, 1 \mathrm{H},-\mathrm{NH}\left(\right.\right.$ exchangeable with $\left.\left.\mathrm{D}_{2} \mathrm{O}\right)\right){ }^{13} \mathrm{C}$ NMR (100 MHz, $\left.\mathrm{CDCl}_{3}\right) \delta 14.3,24.4,24.7,27.8,39.1,46.7,49.2$, 57.2 , 60.7, 61.2, 106.8, 110.1, 111.0, 117.6, 117.9, 119.3, 121.7, $122.3,124.0,125.6,126.7,128.2$, 128.7, 128.9, 129.6, 134.2, 136.5, 138.5, 142.1, 147.21, 150.3, 158.6, 173.1, 183.1 HRMS calcd for $\mathrm{C}_{35} \mathrm{H}_{33} \mathrm{FN}_{6} \mathrm{O}_{4}[\mathrm{M}+1]^{+}$621.2581, found 621.2543.

\section{Cell culturing}

MCF-7 cells were cultured in DMEM media with 10\% FBS and 1\% penicillin-streptomycin and MDA-MB-231 cells were cultured in DMEM supplemented with 5\% FBS and 1\% penicillin-streptomycin, both incubated at $37^{\circ} \mathrm{C}$ and $5 \%$ carbon dioxide. ${ }^{22}$

\section{MTT assay}

Cells were seeded in 96 well plates at a density of 5000 cells per well in triplicate in media. After 24 hours (h), the test compounds, diluted in complete Dulbecco's media Eagle's medium (DMEM) were added to each well. Cells were treated with a range of different concentrations of test compounds $(1 \mu \mathrm{M}, 5 \mu \mathrm{M}, 10 \mu \mathrm{M}, 20 \mu \mathrm{M}, 50$ $\mu \mathrm{M}, 100 \mu \mathrm{M})$ for $24 \mathrm{~h}$ at $37{ }^{\circ} \mathrm{C}$ and $5 \%$ carbon dioxide. Subsequently, sterile $5 \mu \mathrm{L}$ of $5 \mathrm{mg} \mathrm{mL}^{-1} \mathrm{MTT}$ (Sigma-Aldrich) dissolved in PBS was added to each well and incubated with cells for $2 \mathrm{~h}$. Solubilization solution $(10 \% \mathrm{SDS}, 10 \mathrm{mM} \mathrm{HCl})$ of equal volume to the wells was then added to each well, which was incubated with cells for $16 \mathrm{~h}$ at $37^{\circ} \mathrm{C}$. The optical density of each well was read at $570 \mathrm{~nm}$ using a microtiter plate reader (Thermo Fisher Scientific Multiskan GO Microplate Reader, SkanIt ${ }^{\mathrm{TM}}$ software).

\section{Statistical analysis}

The statistical analysis was carried out using Excel® and IC $_{50}$ values were determined using Graph pad Prism5 software (Hearne Scientific Software). The experiments were performed in duplicate and the statistical significance was calculated using student's $t$-test. A $p$-value of less than 0.05 was used to estimate the significance of the observations. A $Z$-factor was calculated for each 96-well plate and assays having $Z$-factor above $>0.5$ were included in the statistical analysis. ${ }^{25}$

\section{Molecular docking}

The X-ray crystal structure of the estrogen receptor- $\alpha$ (PDB ID: 3ERT) was obtained from the RCSB Protein Data Bank (http:// www.rcsb.org/pdb/home/home.do). Using the UCSF Chimera package the ligand and receptor complexes were prepared for docking. ${ }^{26}$ Chem3D Ultra was used to construct a threedimensional structure of the native ligand and most active isatin conjugates. The 3D structure of the compounds was further optimised using the force field, MMFF94s in the Avogadro package (V 1.2.0). AutoDock Tools (V 1.5.6) was used to establish the grid box parameters. All non-polar hydrogens were merged, Gasteiger partial atomic charges added, and rotatable bonds assigned. The grid box of the complex had a grid spacing of $0.375 \AA$. The parameter of the grid points are given in Table 4 $(\mathrm{ESI} \dagger)$. Autodock Vina was used to perform the docking of each ligand into the IGF-1 receptor in triplicate. ${ }^{27}$ The docking systems were validated by redocking the ligand of the protein crystal structures into the binding site. The Protein-Ligand Interaction Profiler (PLIP) was used to analyse the non-polar interactions between the protein and ligand in complex. ${ }^{28}$

\section{Conflicts of interest}

The authors declare no conflict of interest.

\section{Abbreviations}

$\begin{array}{ll}\text { BC } & \text { Breast cancer } \\ \text { TH } \beta C & \text { Tetrahydro- } \beta \text {-carboline } \\ \text { SERMs } & \text { Selective estrogen receptor modulators } \\ \text { ER } & \text { Estrogen receptor } \\ \text { IC }_{50} & 50 \% \text { Inhibitory concentration } \\ \text { SAR } & \text { Structure-activity relationship }\end{array}$

\section{Acknowledgements}

Financial assistance from Council of Scientific and Industrial Research, New Delhi, India, under CSIR-JRF fellowship CSIR Ref No. 09/254(0257)/2016-EMR-I gratefully acknowledged. The 
authors also would like to thank the National Research Foundation of South Africa for their support in facilitating our research (UID: 99563). We would also like to thank the Centre for High Performance Computing based in Cape Town for access to computational resources. MCF7 (The European Collection of Authenticated Cell Cultures (ECACC), UK). MDAMB-231 (The American Type Culture Collection (ATCC), USA).

\section{Notes and references}

1 R. L. Siegel, K. D. Miller and A. Jemal, Ca-Cancer J. Clin., 2018, 68, 7-30.

2 S. Malvia, S. A. Bagadi, U. S. Dubey and S. Saxena, Asia-Pac. J. Clin. Oncol., 2017, 13, 289-295.

3 N. Neuss, M. Gorman and W. Hargrove, J. Am. Chem. Soc., 1964, 86, 1440-1442.

4 L. Garrett, O. Carrier Jr and B. H. Douglas, Eur. J. Pharmacol., 1967, 2, 236-238.

5 P. Jakubec, A. Hawkins, W. Felzmann and D. J. Dixon, J. Am. Chem. Soc., 2012, 134, 17482-17485.

6 P. B. Simon, J. L. Ovenden, C. H. Nielson, R. H. Liptrot, D. M. Willis, A. D. Tapiolas, C. A. Wright and C. A. Motti, Phytochem. Lett., 2011, 4, 69-71.

7 K. B. Wang, Y. T. Di, Y. Bao, C. M. Yuan, G. Chen, D. H. Li, J. Bai, H. P. He, X. J. Hao, Y. H. Pei, Y. K. Jing, Z. L. Li and H. M. Hua, Org. Lett., 2014, 16, 4028-4031.

8 (a) S. Mahale, S. B. Bharate, S. Manda, P. Joshi, P. R. Jenkins, R. A. Vishwakarma and B. Chaudhuri, Cell Death Dis., 2015, 6, 1743; (b) S. Kumar, A. Singh, K. Kumar and V. Kumar, Eur. J. Med. Chem., 2017, 142, 48.

9 H. A. Mohamed, N. M. R. Girgis, R. Wilcken, M. R. Bauer, H. N. Tinsley, B. D. Gary, G. A. Piazza, F. M. Boeckler and A. H. Abadi, J. Med. Chem., 2011, 54, 495-509.

10 C. L. Tourneau, E. Raymond and S. Faivre, Ther. Clin. Risk Manage., 2017, 2, 341.

11 R. J. Motzer, M. D. Michaelson, B. G. Redman, G. R. Hudes, G. Wilding, R. A. Figlin, M. S. Ginsberg, S. T. Kim, C. M. Baum, S. E. De Primo, J. Z. Li, C. L. Bello, C. P. Theuer, D. J. George and B. I. Rini, J. Clin. Oncol., 2006, 24, 16.
12 C. D. Teaux, V. Leblanc, G. B. Langer, S. Parent, E. Asselin and G. B. Rube, Steroids, 2008, 73, 1077-1089.

13 S. Kumar, L. Gu, G. Palma, M. Kaur, A. S. Pillay, P. Singh and V. Kumar, New J. Chem., 2018, 42, 3703.

14 S. Kumar, L. Gu, G. Palma, M. Kaur, A. S. Pillay, P. Singh and V. Kumar, ACS Omega, 2018, 3, 12106-12113.

15 A. Singh, S. T. Saha, S. Perumal, M. Kaur and V. Kumar, ACS Omega, 2018, 3, 5808-5813.

16 S. Kumar, T. bains, A. S. W. Kim, C. Tam, J. Kim, L. W. Cheng, K. M. Land, A. Debnath and V. Kumar, Front. Cell. Infect. Microbiol., 2018, 8, 380.

17 Shalini, A. Viljoen, L. Kremer and V. Kumar, Bioorg. Med. Chem. Lett., 2018, 28, 1309-1312.

18 A. Rani, A. Singh, J. Gut, P. J. Rosenthal and V. Kumar, Eur. J. Med. Chem., 2018, 143, 150-156.

19 H. Song, Y. Liu, L. Wang and Q. Wang, J. Agric. Food Chem., 2014, 5, 1010-1018.

20 M. L. V. Linn and J. M. Cook, J. Org. Chem., 2010, 75, 35873599.

21 X. Fu and J. M. Cook, J. Org. Chem., 1993, 58, 661-672.

22 T. Wang and J. M. Cook, Org. Lett., 2000, 2, 2057-2059.

23 S. Sagar, L. Esau, B. Moosa, N. M. Khashab, V. B. Bajic and M. Kaur, Anti-Cancer Agents Med. Chem., 2014, 14, 170-180.

24 C. D. Savi, R. H. Bradbury, A. A. Rabow, R. A. Norman, C. D. Almeida, D. M. Andrews, P. Ballard, D. Buttar, R. J. Callis, G. S. Currie, J. O. Curwen, C. D. Davies, C. S. Donald, L. J. L. Feron, H. Gingell, S. C. Glossop, B. R. Hayter, S. Hussain, G. Karoutchi, S. G. Lamont, P. MacFaul, T. A. Moss, S. E. Pearson, M. Tonge, G. E. Walker, H. M. Weir and Z. Wilson, J. Med. Chem., 2015, 58, 8128-8140.

25 J. H. Zhang, T. D. Y. Chung and K. R. Oldenburg, J. Biomol. Screening, 1999, 4, 67-73.

26 E. F. Pettersen, T. D. Goddard, C. C. Huang, G. S. Couch, D. M. Greenblatt, E. C. Meng and T. E. Ferrin, J. Comput. Chem., 2004, 25, 1605-1612.

27 O. Trott and A. J. Olson, J. Comput. Chem., 2010, 31, 455-461. 28 S. Salentin, S. Schreiber, V. J. Haupt, M. F. Adasme and M. Schroeder, Nucleic Acids Res., 2015, 43, 443-447. 